\title{
OPEN Actin cytoskeletal inhibitor 19,20-epoxycytochalasin Q sensitizes yeast cells lacking ERG6 through actin-targeting and secondarily through disruption of lipid homeostasis
}

\author{
Kwanrutai Watchaputi ${ }^{1}$, Pichayada Somboon ${ }^{2}$, Nipatthra Phromma-in ${ }^{1}$, \\ Khanok Ratanakhanokchai ${ }^{1}$ \& Nitnipa Soontorngun ${ }^{1 \bowtie}$
}

Repetitive uses of antifungals result in a worldwide crisis of drug resistance; therefore, natural fungicides with minimal side-effects are currently sought after. This study aimed to investigate antifungal property of 19, 20-epoxycytochalasin Q (ECQ), derived from medicinal mushroom Xylaria sp. BCC 1067 of tropical forests. In a model yeast Saccharomyces cerevisiae, ECQ is more toxic in the erg6 $\Delta$ strain, which has previously been shown to allow higher uptake of many hydrophilic toxins. We selected one pathway to study the effects of ECQ at very high levels on transcription: the ergosterol biosynthesis pathway, which is unlikely to be the primary target of ECO. Ergosterol serves many functions that cholesterol does in human cells. ECQ's transcriptional effects were correlated with altered sterol and triacylglycerol levels. In the ECQ-treated $\Delta$ erg 6 strain, which presumably takes up far more ECO than the wild-type strain, there was cell rupture. Increased actin aggregation and lipid droplets assembly were also found in the erg6 6 mutant. Thereby, ECQ is suggested to sensitize yeast cells lacking $E R G 6$ through actin-targeting and consequently but not primarily led to disruption of lipid homeostasis. Investigation of cytochalasins may provide valuable insight with potential biopharmaceutical applications in treatments of fungal infection, cancer or metabolic disorder.

The incidence of the COVID-19 pandemic provides a valuable lesson and increases our awareness on dangerous virus as well as other bugs, including microbes. Microbial drug-resistance is indeed a real threat to the global community and economy. International agencies and experts have predicted that there could be over 10 million deaths yearly by the year 2050, and, ten years from now, antimicrobial resistance could force up to 24 million people at risk of extreme poverty (World Health Organization). The need for prevention and efforts to overcome antimicrobial resistance are now urgently called for. On a rise, invasive fungal infections have caused high morbidity and mortality worldwide, especially in immunocompromised people including AIDS (acquired immune deficiency syndrome) and cancer patients as well as transplant recipients, and even children and senior citizen are also at risk. Mortality rates in critically ill patients are high, ranging from 40 to $70 \%$, depending on the severity of the infections ${ }^{1}$, while few antifungals are available for the treatment of fungal infections. For the past 60 years, among the antifungals available, amphotericin $\mathrm{B}(\mathrm{AmB})$ has been developed and used as the gold-standard antifungal for treatment of invasive pathogenic fungal infections ${ }^{2}$. AmB targets ergosterol, the main sterol component of the fungal cell membrane, possibly through generation of membrane pores that cause leakage of ions and subsequent fungal cell death. However, the administration of AmB is limited because it also interacts with the cholesterol present in human cell membranes and causes severe undesired side effects ${ }^{3}$. The azole antifungals developed in the early 1980s target enzymes of the ergosterol biosynthesis pathways and

\footnotetext{
${ }^{1}$ Division of Biochemical Technology, School of Bioresources and Technology, King Mongkut's University of Technology Thonburi (KMUTT), Bangkok 10150, Thailand. 'Division of Fermentation Technology, Faculty of Food Industry, King Mongkut's Institute of Technology Ladkrabang (KMITL), Bangkok 10520, Thailand. ${ }^{\boxplus}$ email: nitnipa.soo@kmutt.ac.th
} 
display lower toxicity ${ }^{4,5}$. Azoles are therefore commonly used in clinical practice despite their fungistatic effect which eventually leads to the development of drug resistance. Alternatively, lower administered doses of AmB in combination with azoles or other classes of antifungals are often recommended in current antifungal therapies ${ }^{6}$.

The primary mechanisms of action of most antifungal drugs is to disrupt the cellular composition of the fungus and to alter the function of membrane-associated proteins, compromising the integrity of the fungal membrane $e^{7}$. Since the yeast ergosterol and human cholesterol are structurally and functionally similar, the occurrence of multiple adverse reactions may be observed ${ }^{3}$. Biosynthesis of sterols in yeast is a multistep control process. An early, key step is the rate-limiting conversion of 3-hydroxy-3-methylglutaryl coenzyme A (HMG-CoA) to mevalonic acid catalyzed by HMG-CoA reductase, encoded by the HMG1 and $H M G 2^{8}$. These two isoenzymes display different modes of feedback control and regulation ${ }^{8}$. The accumulation of squalene in HMG1-overexpressing strains suggests additional bottlenecks in the postsqualene formation steps ${ }^{9,10}$. In fact, there are overlapping mechanisms for controlling ergosterol biosynthesis. These include transcriptional regulation by the sterol regulatory element transcription factors Upc2 and Emc22, the heme-binding regulator Hap1 and the repressors Rox1 and Mot3 as well as a feedback inhibition of enzymes and changes in their subcellular localization ${ }^{11}$. Enhanced squalene accumulation is shown in a mutant overexpressing mutant Hmg2; however, additional deletion of ERG6 do not further enhance squalene accumulation but transfer surplus squalene into C27 sterols, suggesting a lack of ergosterol feedback inhibition ${ }^{12}$. The synthesis of ergosterol in yeasts is a complex pathway, involving more than 20 distinct reactions. The sterol biosynthesis pathways of ergosterol and cholesterol are highly similar, except for the final steps of the pathways ${ }^{13}$. Disruption of $E R G$ genes in the late step of the ergosterol biosynthesis pathway also results in lower activity of Pdr5p drug efflux transporter and susceptibility to stresses ${ }^{14}$.

Due to the limited availability of antifungal agents for combating an increasing number of fungal infections, the urgent need for new antifungal drugs remains an important public health issue worldwide ${ }^{15}$. Novel antifungals with effective fungicidal activity and specificity for fungal targets are important to fight against drug-resistant fungi. In nature, a variety of filamentous fungi produce bioactive compounds for self-defence, thereby offering potential sources of valuable drug leads, including caspofungin (a semisynthetic compound modified from fungi-derived pneumocandin B0), penicillin, and griseofulvin ${ }^{16}$. In recent years, a plethora of new bioactive secondary metabolites of interest and potential drug leads are derived from filamentous fungi. Xylaria spp., present in tropical forests, have also been reported for application in traditional Chinese folk medicines with potential biological properties such as immune-modulatory, antimalarial, antifungal, antiviral, and anticancer properties $^{7}$. For examples, Xylaria primorskensis produces xylaranic acid with potent antibacterial activity ${ }^{17-19}$ or, Xylaria sp. Acra L38 extract displays antifungal activity and contains medicinal agents such as zofimarin ${ }^{20}$ and griseofulvin, which inhibit fungal plant pathogens ${ }^{21}$. Despite its high potential, there has been little exploration of biopharmaceutical applications for Xylaria spp. Recently, a cultural extract of the fungal Xylaria sp. BCC 1067 was reported to be a potential source of novel antifungal agents ${ }^{22}$. This study aimed to further characterize the antifungal action of 19,20-epoxycytochalasin Q (ECQ), present in the extract of Xylaria sp. BCC $1067^{23}$, in mediating fungal cell death and lipid homeostasis. An alteration in sterol biosynthesis following ECQ treatment was investigated using $S$. cerevisiae wild-type and the $\Delta$ erg6 strains, which the latter lacks a key antifungal target enzyme of ergosterol biosynthesis, as a model for study.

\section{Results}

Antifungal activity of 19,20-epoxycytochalasin $Q$ against the S. cerevisiae ERG deletion strains. Identification of bioactive compounds present in the cultural extract of Xylaria BCC 1067 indicated that 19,20-epoxycytochalasin Q (ECQ) is present, in addition to buformin, a-peltatin, 3-methylhistidine and some acid derivatives such as gluconic acid, 4-guanidinobutanoic acid, gibberellic acid, and picolinic acid. ECQ possess interesting biological activities, including an antibacterial and antiplasmodial property ${ }^{24}$; therefore, it was selected for further investigation. To examine the antifungal action of ECQ, the susceptibility of mutant yeast strains with a deleted gene of the enzyme in the ergosterol biosynthetic pathway was first investigated since many of them are served as antifungal drug targets. The extract of Xylaria sp. BCC 1067 at concentrations ranging from 0 to $2000 \mu \mathrm{g} / \mathrm{ml}$ and a positive control antifungal drug fluconazole, known to target the Erg11p enzyme, were also included. In comparison to the parental BY4742 strain, the $\Delta u p c 2, \Delta e r g 4$, and $\Delta$ erg6 strains showed increased sensitivity to fluconazole, as shown by growth assays and serial dilution spot tests (Fig. 1a,b) as previously reported ${ }^{25}$. Growth of the $\Delta h m g 1$ and $\Delta e r g 28$ strains was slightly inhibited but not that of the $\Delta$ sut 1 and $\Delta$ erg5 strains (Fig. 1a). Interestingly, only the $\Delta$ erg6 strain had increased sensitivity to the Xylaria extract tested when compared to the wild-type $S$. cerevisiae strain, with exhibited $\mathrm{MIC}_{50}, \mathrm{MIC}_{80}$ and MFC values of $375 \mu \mathrm{g} /$ $\mathrm{ml}, 900 \mu \mathrm{g} / \mathrm{ml}$ and 2,000 $\mu \mathrm{g} / \mathrm{ml}$, respectively (Fig. 1c,d). The involvement of Erg6p in conferring resistance to the Xylaria extract was then further investigated using the purified ECQ. Selectively, the $\Delta$ erg6 strain exhibited increased sensitivity to ECQ, with an $\mathrm{MIC}_{50}$ value of $295 \mu \mathrm{g} / \mathrm{ml}$ and an MFC value of $1000 \mu \mathrm{g} / \mathrm{ml}$ (Fig. 1e), which displays slightly better antifungal activity to that observed from the extract of Xylaria. At $1000 \mu \mathrm{g} / \mathrm{ml}$ of ECQ, the $\Delta$ erg6 strain could barely survive, indicating the important role of Erg6p in mediating resistance to antifungal ECQ (Fig. 1f). Additional antifungal assays indicated that ERG6- , ERG1- or ERG11-overexpressing strains show better tolerance to ECQ as compared to the parental strain (Fig. 1g,h). This could be because these ERGoverexpressing strains produce enough or high ergosterol content for maintaining plasma membrane integrity; therefore, they showed ECQ resistance. However, the maximum growth and viability also depended on the level of expression of each gene contained in the plasmid. ERG11 confered the highest ECQ resistance when cell viability was compared at an equal level of gene expression. Here, ERG1 expression level was approximately 40X higher than those of ERG11 and ERG6 genes. 
(a)

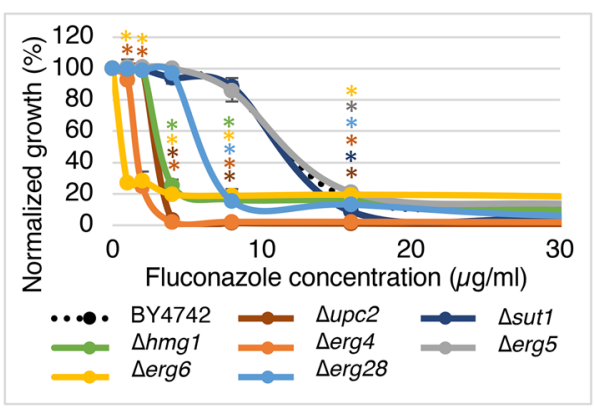

(c)

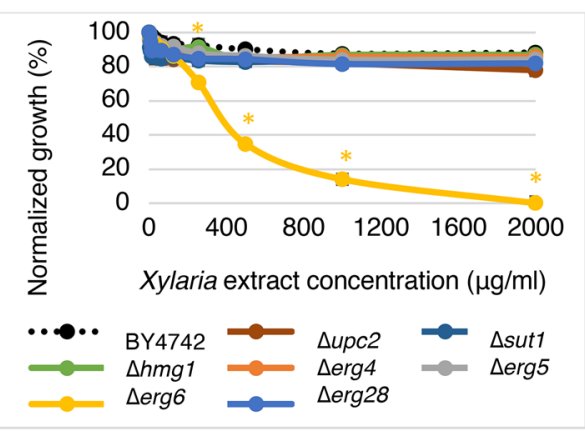

(e)

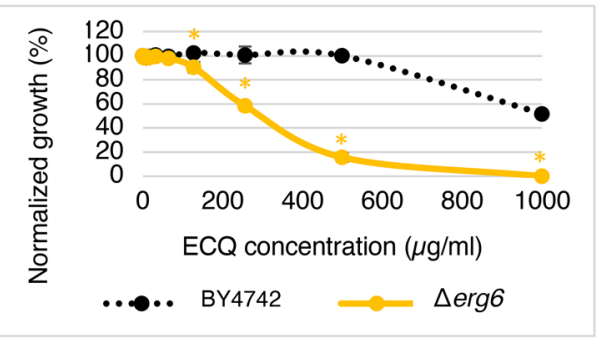

(g)

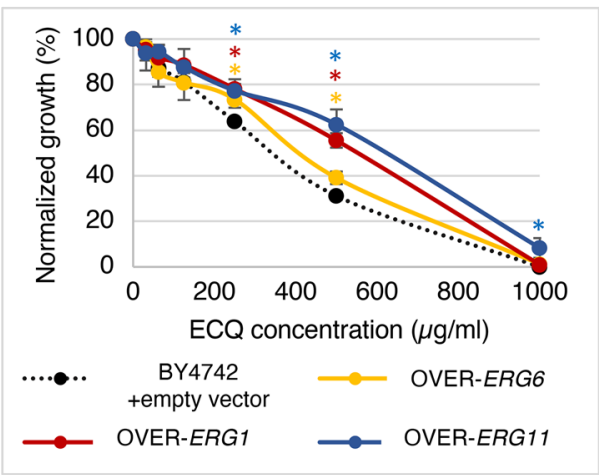

(b)

Fluconazole concentration $(\mu \mathrm{g} / \mathrm{ml})$

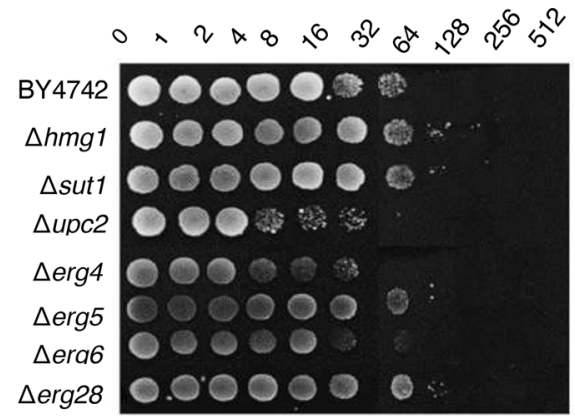

(d)

Xylaria extract concentration $(\mu \mathrm{g} / \mathrm{ml})$

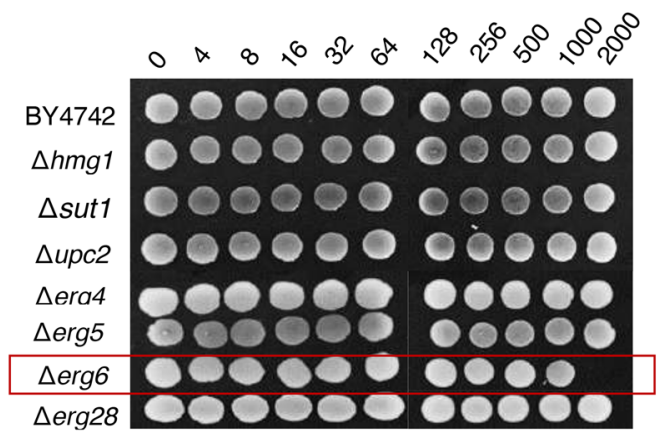

(f)

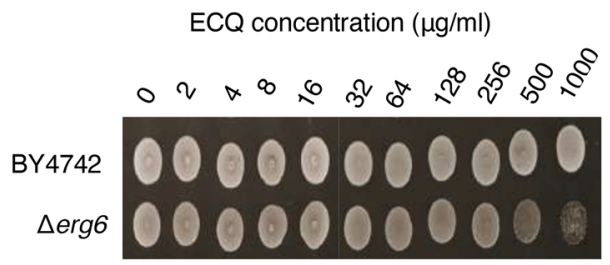

ECQ concentration $(\mu \mathrm{g} / \mathrm{ml})$

(h)

BY4742+

empty vector

OVER-ERG1

OVER-ERGG

OVER-ERG11

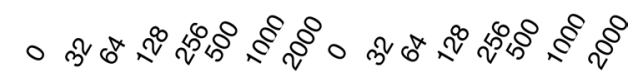

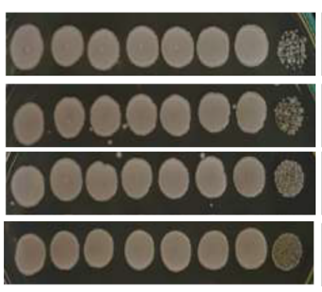

$10^{0}$ dilution

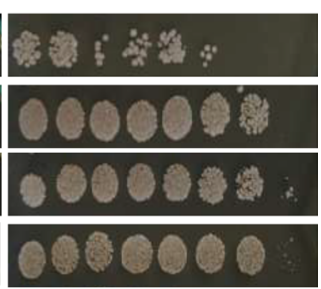

$10^{-3}$ dilution
Fig. 1 Watchaputi et al.

Figure 1. Susceptibility and survival of S. cerevisiae strains with a deletion or overexpression in the gene for the ergosterol biosynthesis during the treatments with different concentrations of fluconazole (a, b), Xylaria extract $(\mathbf{c}, \mathbf{d})$, or ECQ (e-h) using micro-dilution assays and spot tests, respectively. Cells from micro-dilution assays were directly spotted $\left(10^{0}\right.$ dilution) or diluted 1000 times $\left(10^{-3}\right.$ dilution $)$ prior to be spotted on YPD plates. Growth of the overexpression strains were compared with the wild-type strain. ${ }^{\star}$ was referred to the mean difference with significant $p$ value of $<0.05$.

ECQ induced the expression of the fungal-specific ERG6 gene. Previous work has shown that antifungal drugs including ketoconazole induce the expression of many genes, including ergosterol biosynthetic genes, required for conferring drug resistance ${ }^{26-28}$. The qRT-PCR analysis was therefore performed by using 


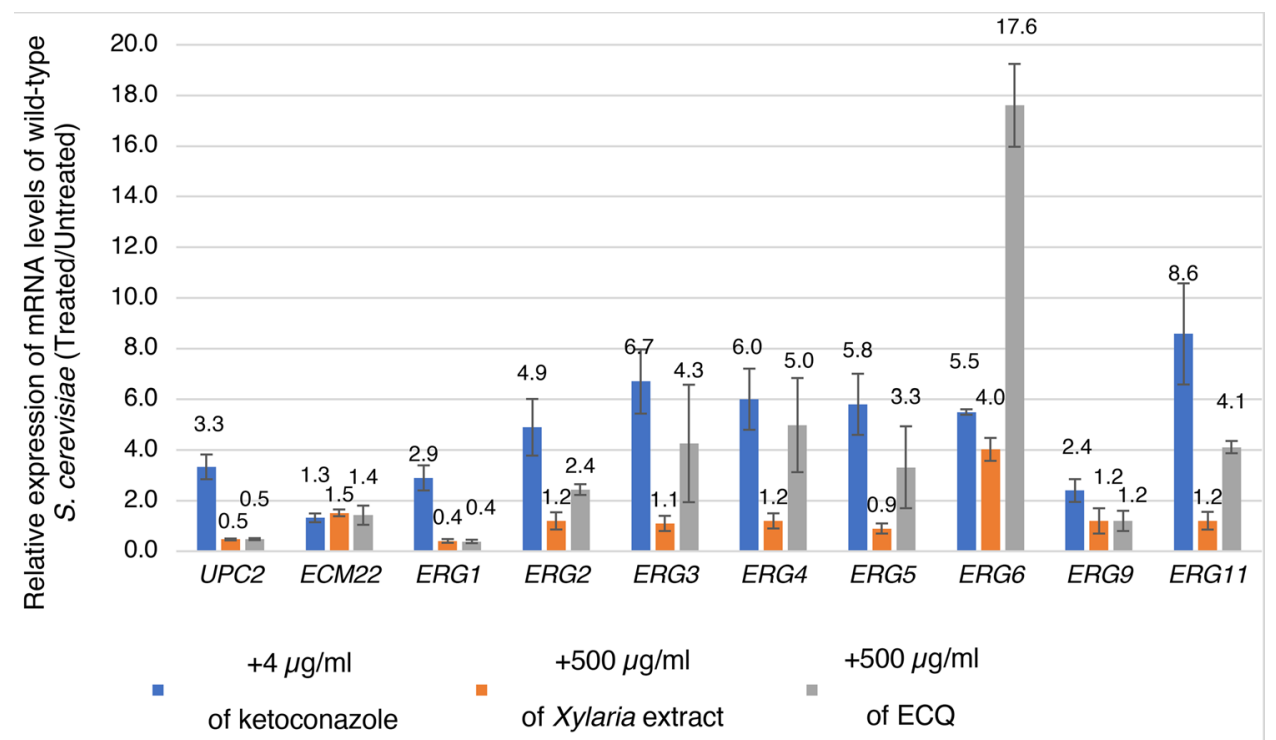

Figure 2. Relative expression of genes in ergosterol biosynthesis. S. cerevisiae BY4742 wild-type strain was treated with $4 \mu \mathrm{g} / \mathrm{ml}$ of ketoconazole, $500 \mu \mathrm{g} / \mathrm{ml}$ of Xylaria extract, or $500 \mu \mathrm{g} / \mathrm{ml}$ ECQ for $2 \mathrm{~h}$. The relative mRNA levels of the treated cells were compared to the untreated cells and normalized with a housekeeping gene TDH3. At least two independent qRT-PCR experiments were performed in triplicates. Error bars represent standard error of the mean (SEM).

TDH3 as a house-keeping gene, and the results confirmed that ketoconazole induces the expression of genes in the ergosterol biosynthetic pathway (ERG11,ERG2, ERG3, ERG4,ERG5, and ERG6) as well as transcription of the regulatory gene UPC2 involved in the up-regulation of these $E R G$ genes (Fig. 2a). Interestingly, the expression of the ERG6 gene was strongly induced by 4.0 -fold and 17.6 -fold after treatment with $500 \mu \mathrm{g} / \mathrm{ml}$ of Xylaria extract or ECQ, respectively (Fig. 2a). The expression of additional ERG genes, namely ERG11, ERG5, ERG4, $E R G 3$, and ERG2, in late ergosterol biosynthesis was also induced by ECQ by 4.1-, 3.3-, 5.0-, 4.3- and 2.4-fold, respectively, when compared to the untreated cells (Fig. 2a). Expression of ERG1 gene was decreased by 2.5 - after induced with Xylaria extract or ECQ (Fig. 2a). Moreover, the expression of UPC2 was also decreased by 2.0 -fold (Fig. 2a) suggested up-regulation of genes in the biosynthesis of ergosterol but not of other pathways under the control of transcription factor Upc2p. Perhaps, ECQ may alter the levels of sterols, such as ergosterol, through alteration of gene expression as a potential feedback mechanism, similar to what has been previously observed for some antifungals ${ }^{28,29}$.

Unusually, the Xylaria extract could not induce expression of other ERG genes except for ERG6, suggesting that ERG6 expression may alternately be in response to impurities (Fig. 2). However, ratio of ECQ in crude Xylaria extract was investigated the by HPLC and found that ECQ appeared approximately $27 \%$ in the extract (Supplementary Fig. 1) which may not contain enough ECQ to induce expression of other ERG genes. It remains to be shown. Nevertheless, further investigation of the role of the ERG6 gene, encoding delta (24)-sterol C-methyltransferase for the conversion of zymosterol to fecosterol of the ergosterol biosynthetic pathway, was initiated. Erg6 is also important for the resistance of cells to antifungal azoles and echinocandins as well as tolerance to acid stress ${ }^{30}$.

ECQ disrupted cellular actin organization and membrane integrity. Cytochalasins are natural bioactive compounds that have been extensively studied. They are known to disrupt actin organization, which has an important role in a variety of cellular processes. During polarized growth, yeast cells contain two types of actin structures which include actin fibre and cortical actin patches. The actin structures play an important role for intracellular transport. Actin fibre acts in the transport of secretory vesicles, whereas actin patches act as ports for the recycle of membrane components from the cortex ${ }^{31}$. Moreover, actin bodies which are F-actin aggregates are observed in stationary-phase cells and inferred to be markers of quiescence ${ }^{32}$. To date, cytochalasins $\mathrm{B}, \mathrm{D}, \mathrm{F}$, and $\mathrm{H}$ have been reported in ability to disrupt actin ${ }^{33}$. The role of cytochalasin Q, namely ECQ to disrupt the organization of the actin $\mathrm{n}$ was recently shown using mutant $S$. cerevisiae $p d r 5$ and Candida cdr 1 strains $^{23}$. Here, the actin organization of $\Delta$ erg6 cells with compromised ergosterol biosynthesis was investigated, using an actin-specific phalloidin-FITC labelled $\operatorname{stain}^{34}$. The wild-type $S$. cerevisiae cells were incubated with concentrations of $1000 \mu \mathrm{g} / \mathrm{ml}$ of the Xylaria extract or $450 \mu \mathrm{g} / \mathrm{ml}$ of ECQ, while the $\Delta$ erg6 cells were incubated with concentrations $450 \mu \mathrm{g} / \mathrm{ml}$ or $225 \mu \mathrm{g} / \mathrm{ml}$, respectively. The results showed that two types of actin structures, including cortical actin patches (blue arrow) and actin fibre (red arrow), formed at the bud site and within the cell, indicating normal actin organization in the untreated wild-type and the $\Delta$ erg6 strains at $2 \mathrm{~h}$ (Fig. 3a,b). At $24 \mathrm{~h}$, yeast cells were going to the stationary phase, leading F-actin to aggregate and to form actin body structure (yellow arrow) as shown in Fig. 3a,b. After Xylaria extract treatment, no apparent change in actin fibre formation 
(a)

Wild-type S. cerevisiae

Untreated

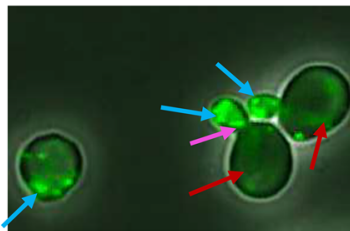

$24 h$
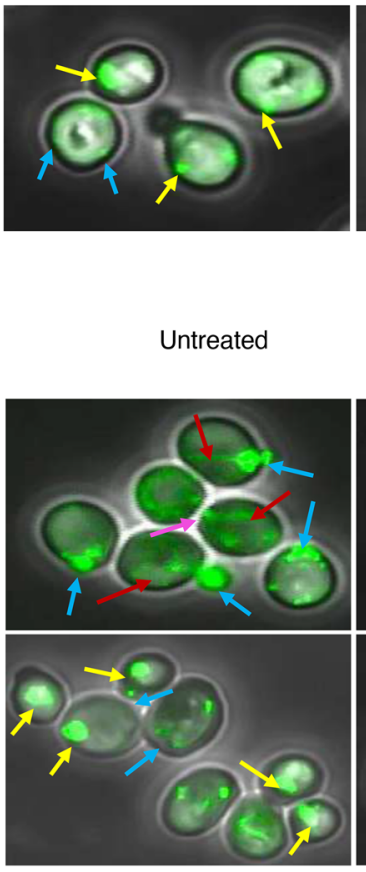

$+1000 \mu \mathrm{g} / \mathrm{ml}$

of Xylaria extract
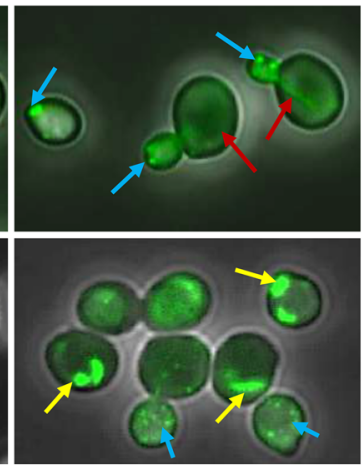

The $\Delta$ erg6 strain

$+450 \mu \mathrm{g} / \mathrm{ml}$

of Xylaria extract

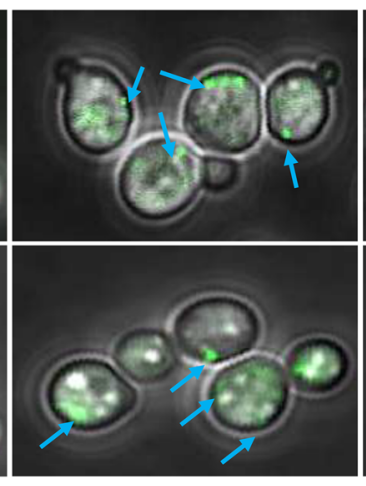

$+450 \mu \mathrm{g} / \mathrm{ml}$ of ECQ
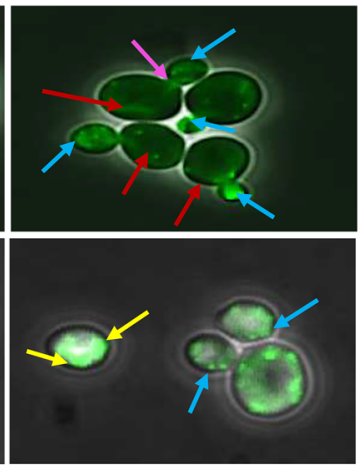

$+225 \mu \mathrm{g} / \mathrm{ml}$ of ECQ

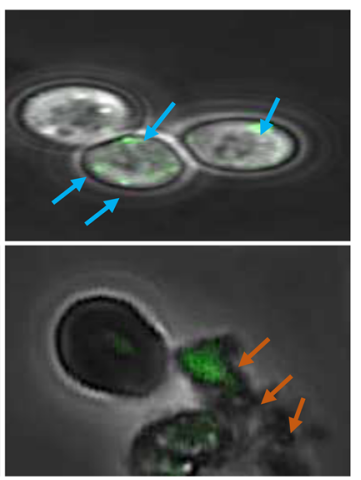

\begin{tabular}{|c|c|c|c|c|c|c|c|}
\hline & \multirow{2}{*}{$\begin{array}{c}\text { Time point } \\
\text { (h) }\end{array}$} & \multicolumn{2}{|c|}{ Untreated } & \multicolumn{2}{|c|}{+ Xylaria extract } & \multicolumn{2}{|c|}{$+\mathrm{ECQ}$} \\
\hline & & Wild-type & $\Delta e r g 6$ & Wild-type & $\Delta e r g 6$ & Wild-type & $\Delta e r g 6$ \\
\hline \multirow[t]{2}{*}{ Actin fibre } & $\longrightarrow 2$ & $46 \pm 0$ & $41 \pm 0$ & $44 \pm 4$ & $0 \pm 0^{*}$ & $42 \pm 1^{*}$ & $0 \pm 0^{*}$ \\
\hline & 24 & $8 \pm 5$ & $0 \pm 0$ & $3 \pm 2^{*}$ & $0 \pm 0$ & $2 \pm 2^{*}$ & $0 \pm 0$ \\
\hline \multirow[t]{2}{*}{ Cortical actin patch } & $\longrightarrow 2$ & $42 \pm 4$ & $45 \pm 1$ & $45 \pm 1$ & $100 \pm 0^{*}$ & $44 \pm 2$ & $100 \pm 0^{*}$ \\
\hline & 24 & $48 \pm 2$ & $21 \pm 4$ & $51 \pm 2$ & $100 \pm 0^{*}$ & $50 \pm 1$ & $0 \pm 0^{*}$ \\
\hline \multirow[t]{2}{*}{ Contractile ring } & $\longrightarrow 2$ & $12 \pm 4$ & $14 \pm 1$ & $6 \pm 1^{*}$ & $0 \pm 0^{*}$ & $9 \pm 0^{*}$ & $0 \pm 0^{*}$ \\
\hline & 24 & $0 \pm 0$ & $0 \pm 0$ & $0 \pm 0$ & $0 \pm 0$ & $0 \pm 0$ & $0 \pm 0$ \\
\hline \multirow[t]{2}{*}{ Actin body } & $\longrightarrow 2$ & $0 \pm 0$ & $0 \pm 0$ & $5 \pm 4^{*}$ & $0 \pm 0$ & $5 \pm 0^{*}$ & $0 \pm 0$ \\
\hline & 24 & $44 \pm 3$ & $79 \pm 4$ & $46 \pm 2$ & $0 \pm 0^{*}$ & $48 \pm 3^{\star}$ & $0 \pm 0^{*}$ \\
\hline
\end{tabular}

Fig. 3 Watchaputi et al.

Figure 3. Effect of Xylaria extract and ECQ on actin cytoskeleton organization (a) and cell membrane integrity (b) in the wild-type S. cerevisiae and the $\Delta$ erg6 strains. Blue arrows indicated cortical actin patches; red arrows indicated actin fibre; yellow arrows indicated actin body; and orange arrows indicated cell breakage. Error bars represent standard error of the mean (SEM) $\left({ }^{*} p<0.05\right.$, using one-way ANOVA compared to the untreated condition). 
(b)

Wild-type S. cerevisiae

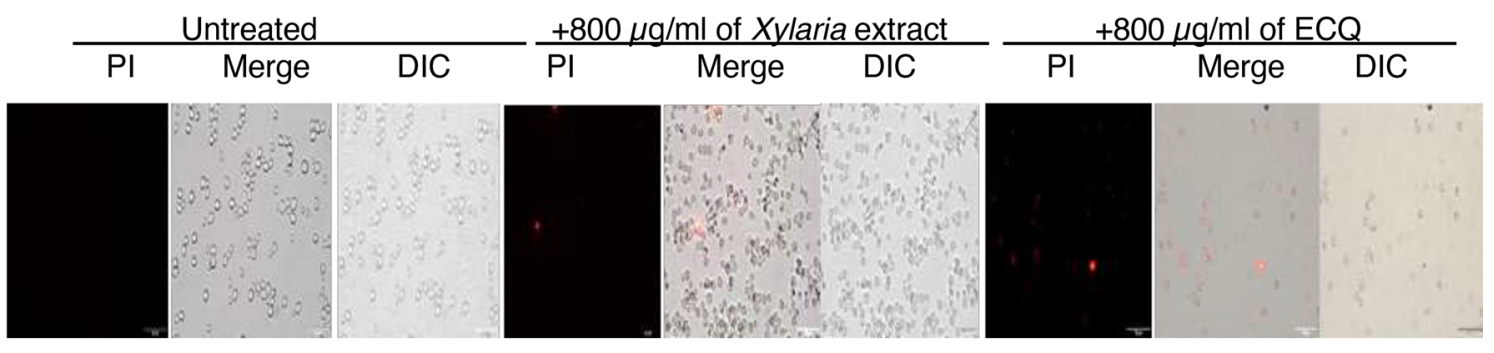

\begin{tabular}{|l|c|c|}
\hline \multicolumn{3}{|c|}{ PI Positive (\%) } \\
\hline $0.65 \pm 1.12$ & $2.77 \pm 0.27$ & $88.48 \pm 7.74^{*}$ \\
\hline
\end{tabular}

The $\Delta$ erg6 strain

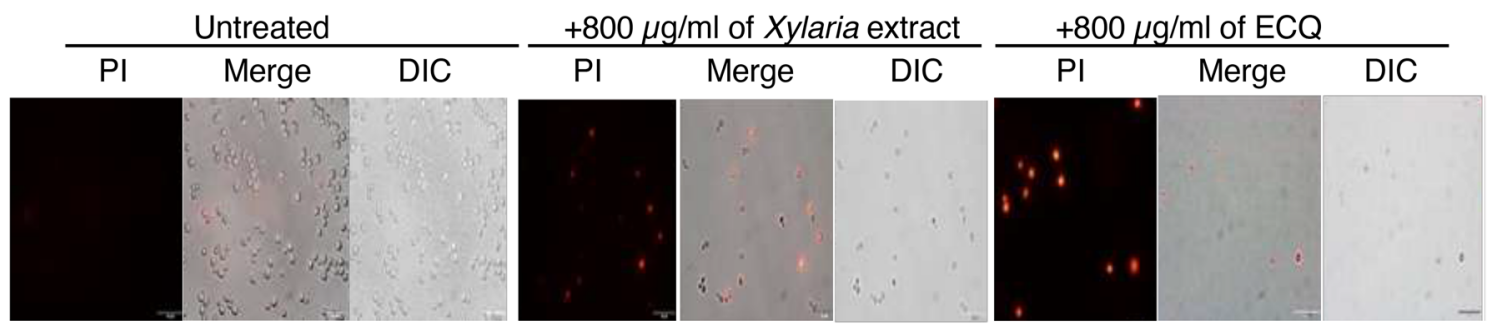

\begin{tabular}{|l|c|c|}
\hline \multicolumn{3}{|c|}{ PI Positive (\%) } \\
\hline $0.94 \pm 0.81$ & $73.72 \pm 6.82^{*}$ & $97.62 \pm 4.12^{\star}$ \\
\hline
\end{tabular}

Figure 3. (continued)

was observed in the wild-type strain, while the actin fibre disappeared in the $\Delta$ erg6 strain at all indicated time points, suggesting disruption of actin polymerization in the cytosol of the $\Delta$ erg6 strain (Fig. $3 \mathrm{a}, \mathrm{b}$ ). For treatment with ECQ, disruption of actin organization was observed after treatment at $24 \mathrm{~h}$ in the wild-type and as early as $2 \mathrm{~h}$ in the $\Delta$ erg6 strain (Fig. 3a,b). ECQ disrupted organization of actin and, this effect of actin aggregation was more obvious in the erg6 $\Delta$ strain that likely takes up more drug and more prone to ECQ toxicity.

Moreover, cell membrane breakage was observed in the $\Delta$ erg6 strain after treatment with ECQ. As shown, treatment with ECQ at $24 \mathrm{~h}$ resulted in damaged cell membranes, membrane rupture, and possibly leakage of the cellular contents of the $\Delta$ erg6 strain (Fig. 3b). The possibility that ECQ may disrupt plasma membrane integrity was tested. Polar fluorescent propidium iodide (PI) stain (MW $=668.4 \mathrm{Da}$ ), which binds to nucleic acids but does not permeate through the membrane, was used to score the number of cells in a population with lost cellular integrity ${ }^{35}$. The untreated $S$. cerevisiae wild-type and the $\Delta$ erg6 strains exhibited a non-PI-positive signal with less than $1.0 \%$ (Fig. 3a,b). However, the Xylaria extract or the ECQ-treated wild-type strain and especially the $\Delta$ erg6 strain exhibited a strong red fluorescent signal, positive for PI staining, which accounted for $2.77 \%$ and $88.48 \%$ of total cells for the wild-type and $73.72 \%$ and $97.62 \%$ of total cells for the $\Delta$ erg6 strain, respectively (Fig. 3a,b). Overall, ECQ interferes with actin organization and causes defective cell morphology, resulting in plasma membrane breakage and cell death.

ERG6 deletion or ECQ treatment altered the sterol composition of yeast cells. A diagram represented the mevalonic pathway and the ergosterol biosynthesis was depicted to include antifungal drug key target enzymes, sterols and responsible transcription factors (Fig. 4a). In yeast, the early step is initiated by acetylCoA to produce an important intermediate, farnesyl pyrophosphate (FPP) ${ }^{36}$. Therefore, mutations during this step of the pathway are lethal. The late step of ergosterol biosynthesis is dedicated to the conversion of FPP to the sterol precursor squalene, the first specific intermediate for the production of ergosterol, mediated by Erg9p. The next step is conversion of squalene to lanosterol, the first sterol molecule of the ergosterol biosynthetic pathway that is mediated by the enzymes Erg1p (squalene epoxidase) and Erg7p (lanosterol synthase) ${ }^{14}$. Conversion of lanosterol to ergosterol requires many enzymes, including Erg11p and Erg6 $\mathrm{p}^{37}$. Deletion of genes in ergosterol biosynthesis alters the sterol and lipid composition of the yeast cell membrane ${ }^{38}$. As shown, the $\Delta \operatorname{erg} 6$ strain is unable to catalyse C-24 methylation, leading to accumulated zymosterol ${ }^{39}$. Lacking of ergosterol biosynthetic enzymes support a critical role for ergosterol in yeasts and fungi ${ }^{40}$. In addition, when Erg $11 \mathrm{p}$ is inhibited, a pathway for alternative sterol biosynthesis that leads to 14 methylergosta 28-24-28 dienol, is activated by treatment 
(a)

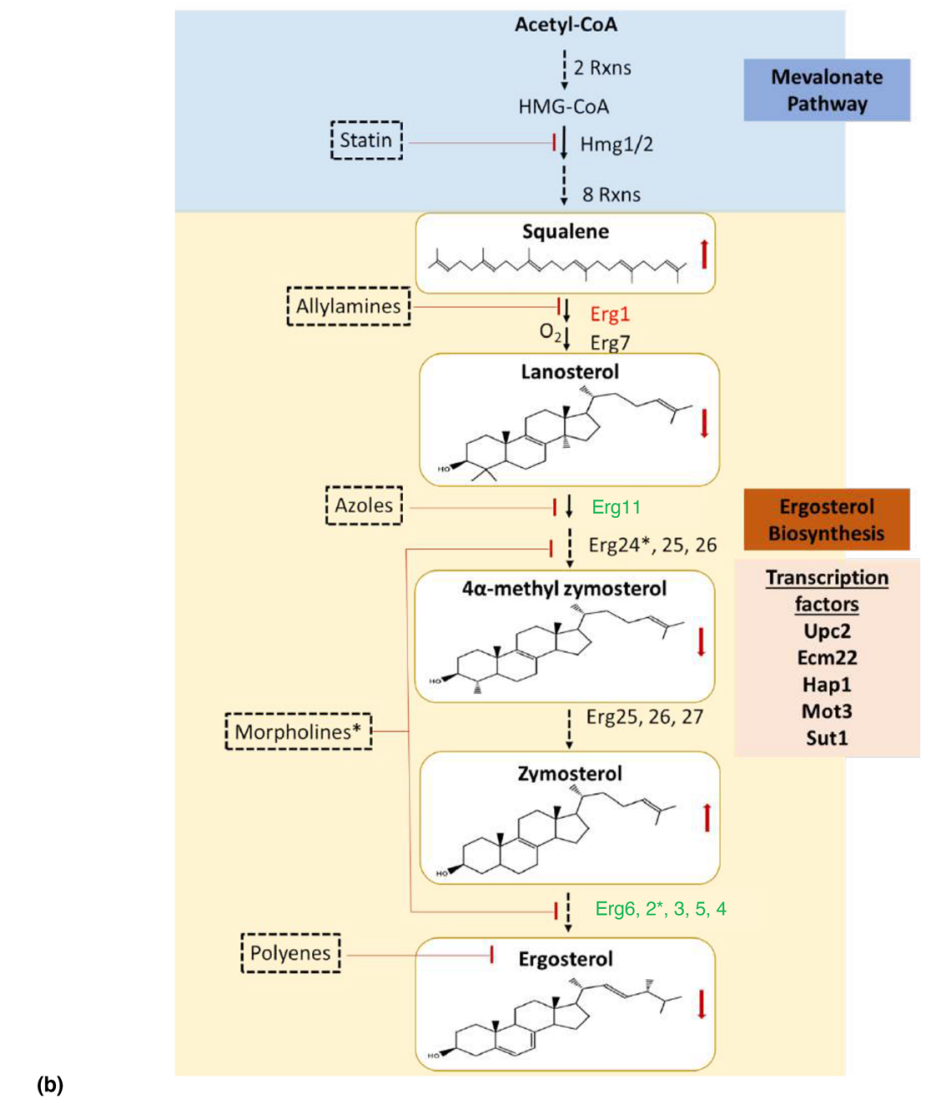

(b)

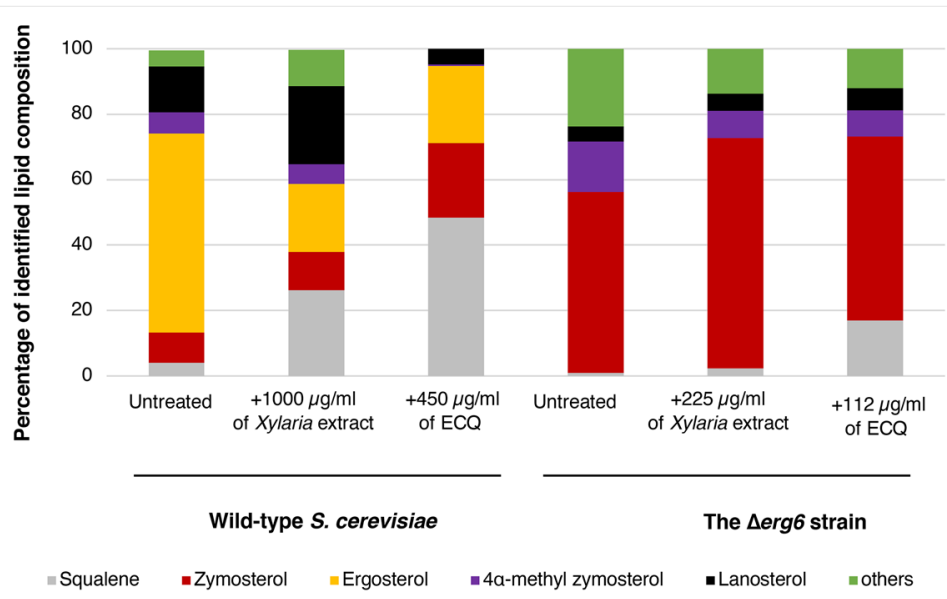

Figure 4. Alteration of sterol and TAG levels as well as formation of LDs clustering were examined during the ECQ treatment in S. cerevisiae. (a) ergosterol biosynthesis pathway in S. cerevisiae, including involved transcriptional factors and targets of antifungal drugs was depicted (b) percentage of identified sterol composition as quantified by GC-MS, (c) LDs formation and Nile Red fluorescent intensity, and the chromatograms of the change in (d) sterol composition and (e) TAG content of yeast cells after treatment with ECQ of S. cerevisiae wild-type and $\Delta$ erg6 strains treated with the Xylaria extract or ECQ. Gene labelled in green or red colour indicated up- or down-regulated expression, respectively. Asterisks indicated target of Morpholines. Red arrows indicated increased or decreased accumulation of sterol level, following the ECQ treatment. "ND." Was referred to "not detected". Error bars represent standard error of the mean (SEM). Different letters above the error bars $(\mathbf{a}-\mathbf{f})$ indicate significant differences at $p$ value of $<0.05$. 
(c)

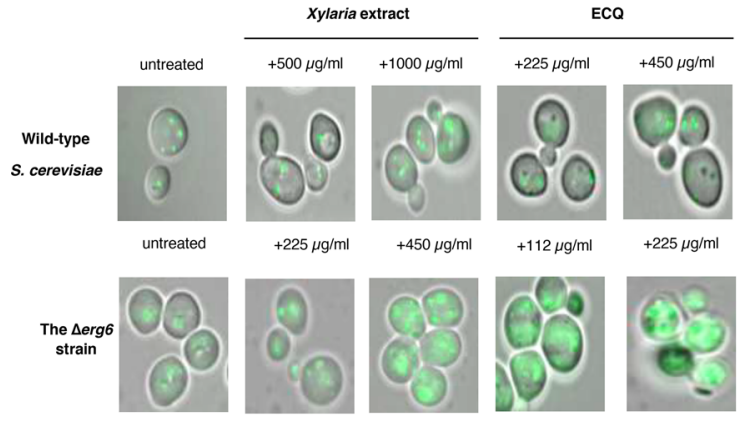

(d)

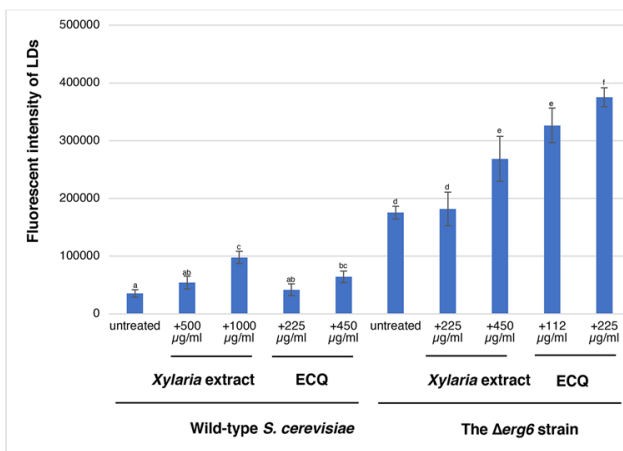

(e)

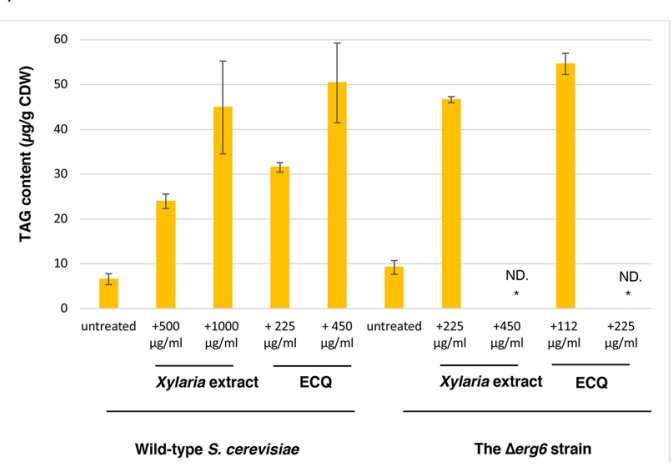

Figure 4. (continued)

with antifungal azoles ${ }^{41}$. Likewise, treatment with ECQ induced cellular feedback through reprogramming the expression of many ERG genes, particularly ERG6 (Fig. 2). Consequently, the alteration of sterol composition in the membrane may be resulted. Using gas chromatography-mass spectrometry (GC-MS), the sterol composition of the wild-type and the erg6 $\Delta$ strains treated with ECQ was examined.

The GC-MS results showed that ergosterol is the main sterol component of the wild-type $S$. cerevisiae cell membrane in the untreated cells, along with the presence of squalene, zymosterol, $4 a$-methyl zymosterol, lanosterol and others (Fig. 4b). Treatment with the Xylaria extract disrupted normal ergosterol biosynthesis as shown by reduced ergosterol content of more than $60 \%$ of the total sterol fraction and increased accumulation squalene, lanosterol, zymosterol and others (Fig. 4b). In the wild-type strain under ECQ treatment, the ergosterol level was also reduced by approximately $60 \%$ compared to the total sterol fraction of the untreated cells (Fig. 4b). ECQ's transcriptional effects on this ERG pathway were matched by alterations to levels of sterol and of triacylglycerol, but it is not possible to determine if the primary effect on lipid levels is transcriptional or not.

Remarkably, over an $1100 \%$ increase in squalene of the total sterol fraction was observed (Fig. 4b). Squalene has been reported to be a toxic lipid species in yeast; excess squalene is normally stored in LDs to prevent its toxicity ${ }^{42,43}$. In fact, the wild-type cells remain viable even with elevated squalene, suggesting normal squalene storage. Regarding the $\Delta$ erg6 strain, an alteration in the sterol profile was dramatically observed (Fig. 4b). Strikingly, the ergosterol level was undetected, but a high accumulation of zymosterol and $4 \alpha$-methyl zymosterol was observed, in comparison to the total sterol fraction of this deletion strain (Fig. 4b). When compared to untreated condition, treatment with the Xylaria extract or ECQ resulted in increasing levels of zymosterol by $27 \%$ and $2 \%$ of the total sterol fraction of the $\Delta$ erg6 strain (Fig. 4b), respectively. Level of toxic squalene in the untreated $\Delta \operatorname{erg} 6$ strain increased by $100 \%$ and $600 \%$ of the total sterol fraction after treatment with the Xylaria extract or ECQ, respectively, thereby explaining increased sensitivity of the ECQ-treated $\Delta$ erg6 strain (Figs. 1, 4b).

Treatment with ECQ enhanced clustering of LDs and accumulation of triacyglycerol (TAG). Lipid droplets (LDs) are versatile organelles with vital roles in lipid metabolism and energy homeostasis in all eukaryotes. LDs biogenesis occurs at specific sites in the ER, in response to excess amounts of lipids or nutrients, and oxidative stress ${ }^{44}$. In normal conditions, wild-type cells produce small LDs that are loosely circulated in cells (Fig. 4c). After treatment with Xylaria extract or ECQ, the wild-type yeast cells showed aggregation of LDs and increased Nile Red fluorescent intensity detection when compared with the untreated cells (Fig. 4c). Nile Red staining and fluorescence microscopy are commonly used to examine cellular neutral lipids, comprising of LDs. This suggested increased accumulation of toxic lipids inside the cells. The $\Delta$ erg6 strain also exhibited LDs aggregation with aberrant Nile Red fluorescent intensity detection when compared to the wild-type strain, suggesting increased accumulation of toxic sterols (Fig. 4b, c). Interestingly, treatment with Xylaria extract or ECQ showed strong Nile Red fluorescent intensity of many big clusters or fusion of LDs, suggesting a mobility block of LDs filled with excessive toxic lipid squalene within the $\Delta \operatorname{erg} 6$ cells (Fig. 4b,c). The results showed not 
only significant lipid droplet clustering which arbitrarily is defined as an aggregation of six or more lipid droplets as previously shown in the $\Delta$ erg1 strain with defective in sterol biosynthesis ${ }^{45}$.

Formation of LD cluster agreed well with the repression of ERG1 expression by ECQ in the wild-type strain (Figs. 2, 4c). The observed fusion of LDs was also found following the striking formation of supersized LDs and clusters of multiple small LDs in the $\Delta$ erg6 strain (Fig. 4c) as shown for the yeast mutant $\Delta$ fld 1 lacking a human seipin homolog, implicated in congenital lipodystrophy ${ }^{46}$. Interestingly, previous work demonstrates that LD biogenesis sites at ER subdomains containing Fld1, Nem1, Yft2, and Pex30 define sites for the localization of the TAG-synthase and droplet formation ${ }^{47}$. Consequently, Erg6 and Pet10 bind to newly formed LDs from the cytosolic side. As a result, deletion of ERG6 caused a devastation in proper LD biogenesis which is critical for cellular function and response to lipotoxicity. Furthermore, the replacement of ergosterol by possibly zymosterol in the plasma membrane of yeast cells ${ }^{48}$ exacerbated the effect of squalene toxicity displayed by the antifungal ECQ, supporting increased sensitivity of in the $\Delta$ erg6 strain, following the ECQ treatment (Figs. 1, 4). Accumulation of free toxic sterol inside the yeast $\Delta$ erg6 strain may exacerbate the effect of ECQ-mediated inhibition of actin cytoskeleton.

Moreover, LDs are cellular organelle involved in storage of metabolic energy in the form of neutral lipids such as sterol esters and triacylglycerol (TAG) ${ }^{49}$. Increased accumulation of lipid droplets was induced by Xylaria extract or ECQ treatment (Fig. 4d). Following treatments with the Xylaria extract or ECQ, in the wild type cells, TAG content was increased in dose-dependent manner with increasing concentrations of Xylaria extract or ECQ from 500 to $1000 \mu \mathrm{g} / \mathrm{ml}$ or 225 to $450 \mu \mathrm{g} / \mathrm{ml}$, respectively (Fig. $4 \mathrm{~d}$ ). While in the $\Delta$ erg6 strain, high accumulation of TAG occurred after treatment with lower concentrations of Xylaria extract or ECQ at 225 or $112 \mu \mathrm{g} / \mathrm{ml}$, respectively (Fig. $4 \mathrm{~d}$ ). Since increasing concentrations of Xylaria extract or ECQ caused dramatic growth inhibition of the $\Delta$ erg6 cells, this resulted in low numbers of cells not enough for TAG detection. Thus, ECQ treatment not only affect sterol composition but also resulted in accumulation of neutral lipid TAG that is correlated with increased numbers and size of LDs (Fig. 4d). It remains to be shown whether ECQ action on actin disruption affects LD mobility.

\section{Discussion}

Antimicrobial drug resistance is presently a worldwide important health concern. Using the model yeast $S$. cerevisiae, a role of antifungal 19,20-epoxycytochalasin Q (ECQ) as a novel sterol modulator and its mechanism of actions are summarized (Fig. 5). Noticeably, varied drug concentrations used in each assay are determined according to cell growth/survivability of wild-type and $\operatorname{erg} 6 \Delta$ strains. The range of ECQ concentrations required for detection of certain cellular events of a variety of experiments, varied between 0 and $1000 \mu \mathrm{g} / \mathrm{ml}$, is dependent on the strain's genetic backgrounds. The concentrations used are adjusted, according to the $\mathrm{MIC}_{80}$ of drugs or the extract to provide comparable numbers of cells in each experimental tested condition. Alternatively, if possible, results of each assay operated by overlapping concentrations of drugs are shown in parallel. For this reason, the relationships between the observed cellular events shall be considered conditionally. Nevertheless, the data on genetical, metabolic or morphological changes could be discussed when the concentrations of ECQ used were comparable. To summarize, treatment of the wild-type strain with ECQ at $500 \mu \mathrm{g} / \mathrm{ml}$ caused up-regulation of some ERGs genes but down-regulation of ERG1 and UPC2 genes (Fig. 2). At similar ECQ concentration of $450 \mu \mathrm{g} / \mathrm{ml}$, in the wild-type strain, disruption of actin organization was slightly observed as shown by aggregated actin fiber and increased accumulation of actin bodies (Fig. 3). Moreover, sterol homeostasis was disrupted as shown by decreased ergosterol content, increased squalene and zymosterol content and increased LDs aggregation with increased accumulation of TAG content (Fig. 4). However, more impaired metabolic and morphological effects were observed in the $\Delta e r g 6$ strain at even lower ECQ concentrations used. At $225 \mu \mathrm{g} / \mathrm{ml}$ of ECQ, disruption of actin organization occurred faster as shown by aggregated actin fiber and increased accumulation of actin patches and actin bodies at $2 \mathrm{~h}$ (Fig. 3). At this ECQ concentration, cells began to die and, there were not enough cells for recovery of sterol or TAG (data not shown). Thus, a lower ECQ concentration of $112 \mu \mathrm{g} / \mathrm{ml}$ was used. Increased squalene and TAG accumulation as well as increased LDs aggregation were evidently found as compared to the untreated condition (Figs. 3 and 4). More severe morphological effect was observed at $24 \mathrm{~h}$ of treatment as shown by disrupted actin structure (Fig. 3). Cell membrane breakage was evidently observed in the wild-type and the $\Delta$ erg6 strains at $800 \mu \mathrm{g} / \mathrm{ml}$ of ECQ treatment (Fig. 3) although this may happen already at lower ECQ concentrations. Overall, results indicated that there was a correlation between genetical, metabolic and morphological changes of cells in response to ECQ treatments (Fig. 5).

Here, some important aspects on molecular mechanisms and cellular responses to ECQ could be drawn. First, several lines of evidence support the requirement of some Erg enzymes including Erg6p, in the adaptive cellular response to ECQ, as shown by the increasing tolerance of these $E R G$-overexpressing strains and altered expression levels upon ECQ treatments (Figs. 1,2). Interestingly, gene expression analysis of ECQ treated wild-type yeast cells showed down regulation of UPC2 and ERG1 genes but not ECM22 which is opposite to regulation of other ERG genes tested (Fig. 2). In sterol-rich and aerobic condition, the basal ERG gene expression is mostly maintained by Ecm22p and Hap1 $p^{11}$. However, under ergosterol depletion as in the case of ECQ-treated cells, $\mathrm{Upc} 2 \mathrm{p}$ is a key regulatory protein to activate the transcription of $E R G$ genes and its own expression ${ }^{11}$. This may explain differential regulation observed in the gene expression analysis. Secondly, adaptive cellular response to the actin inhibitors or ECQ may operate to prevent activation of the whole ergosterol biosynthetic pathway or through selective regulation at some specific ERG promoters or at the first step of pathway via repression of UPC2 and ERG1 expression, respectively, in order to fine-tune lipid composition and function. Such feedback mechanisms may be possible for a following reason. Firstly, ERG1 is essential under aerobic growth conditions, but erg1 null mutants are viable when grown under anaerobic conditions in the presence of ergosterol ${ }^{50}$. Treatment with ECQ reduced expression of ERG1 transcripts by approximately threefold (Fig. 2). Reduced expression 
(a) Wild-type S. cerevisiae

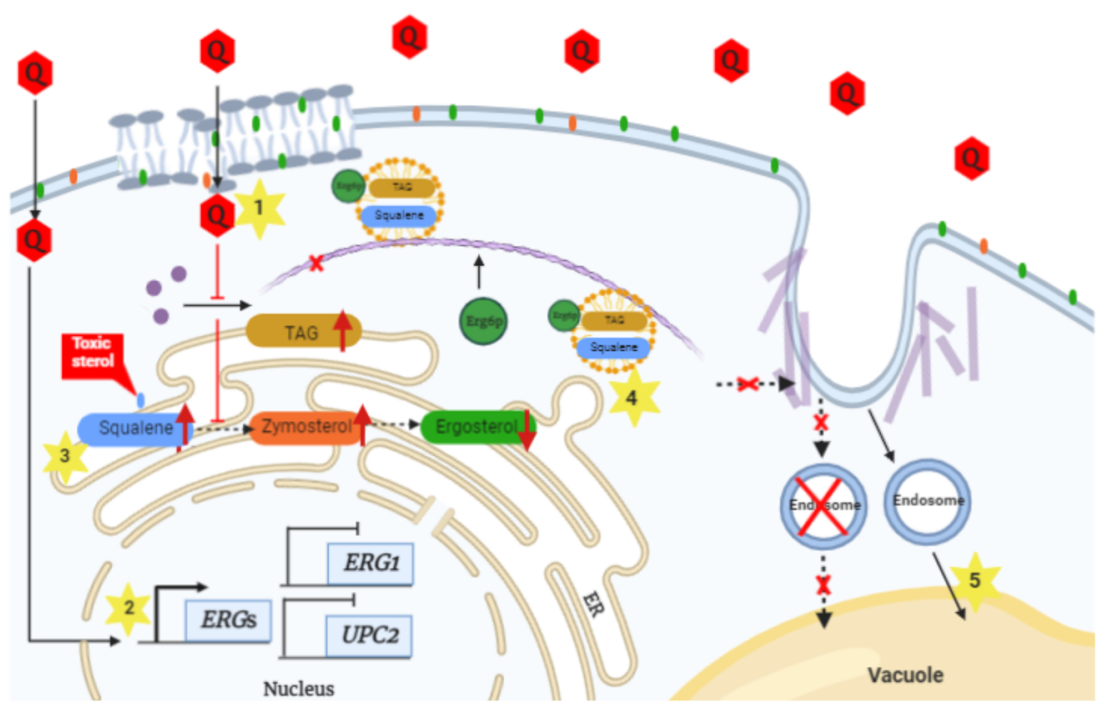

(b) The $\Delta$ erg6 strain
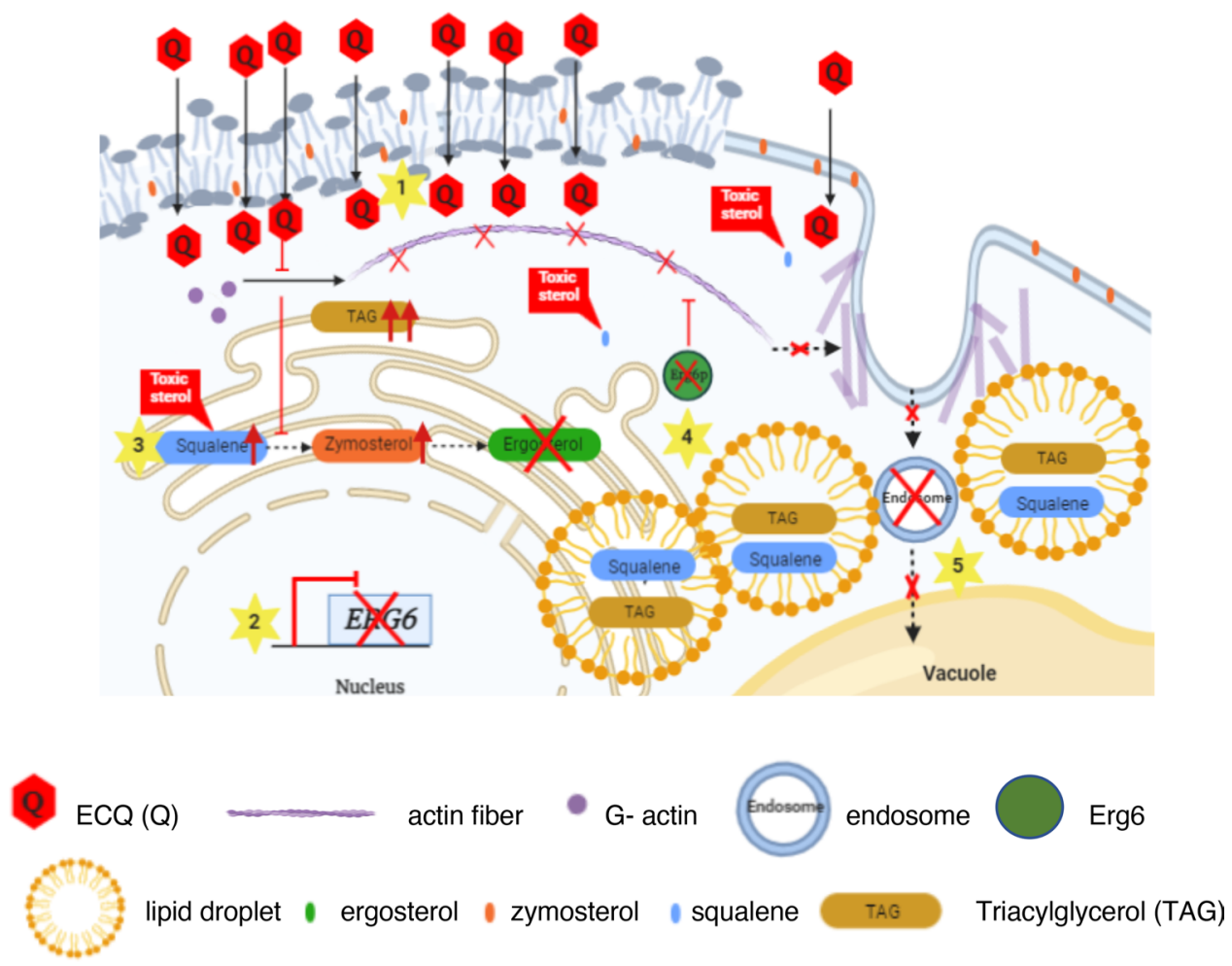

Figure 5. Mode of action of antifungal ECQ in the model yeast S. cerevisiae. (a) In wild-type strain, once entered the cells (1), ECQ not only disrupted actin cytoskeleton organization but also strongly induced expression of $E R G$ genes, including ERG6, to compensate for lower ergosterol level (2). ECQ treatment resulted in the alteration of lipid composition (3), leading to increased accumulation of TAG as well as zymosterol and toxic sterol squalene that normally being stored in LDs. LDs formation was induced (4) while some aggregated actin and damaged protein were also removed via endosomes (5) to reduce effect of ECQ cytotoxicity. (b) In contrast to the $\Delta$ erg6 strain, membrane permeability was increased (1), allowing penetration of drugs including ECQ. Loss of Erg6 caused pronounced effects on ECQ-mediated inhibition of actin cytoskeleton organization and function (2) and alteration of ergosterol metabolism (3), resulting in elevated levels zymosterol, the defective plasma membrane and abnormal cell morphology. Additionally, despite formation of LDs, they were clustered and non-functional (4), resulting in increased accumulation of toxic squalene. However, newly formed LDs could not be properly destined for vacuolar degradation as a result of ECQ inhibition on actin transport of LDs (5). Overall, ECQ displayed a coupling antifungal mechanism, resulting in abnormal actin cytoskeleton organization and altered sterol and lipid homeostasis. Red arrows indicated a decreased or increased of cellular sterol or TAG levels after treatments with ECQ. Yellow star number 1-5 indicated proposed cellular events during ECQ treatments. 
of ERG1 may affect amount of Erg1p or its enzymatic function which is required for proper growth and cell survival under aerobic condition. Thereby, cells may mechanistically respond through ECQ-mediated ERG1 repression by activating the downstream ergosterol biosynthetic genes to enhance ergosterol production. Also, repression of $E R G 1$ has been shown to cause abnormal lipid particle morphology and accumulation of elevated levels of squalene ${ }^{45}$. Thus, activation of genes in the latter steps of ergosterol biosynthesis may be required for controlling level of excess intracellular squalene and maintain lipid homeostasis.

Second, this study reveals the ability of natural antifungal ECQ of the medicinal mushroom Xylaria spp. to aggregate actin and to alter biosynthesis of key components of fungal plasma membrane TAG and sterol in yeast cells (Figs. 3, 4). Despite, there is less effect on sterol composition in the $\operatorname{erg} 6 \Delta$ strain. This indicates that changes of sterol in wild-type cells, at high ECQ concentrations, may be quite indirect. Likely, it may be via a non-sterol target for ECQ, and possibly for extract, which has followed on effects on sterol as shown by the accumulation of LDs (Fig. 4). Thirdly, increased formation of LDs clustering is found following ECQ treatment, especially in the absence of Erg6p (Fig. 4). A mechanism of ER-sterol misplacement mediated through actin cytoskeleton on non-vesicular lipid transport through Erg6-assisociated LDs is suspected to occur during the ECQ treatments (Fig. 5). Defective actin depolymerization and disrupted lipid homeostasis are evident in the $\Delta$ erg6 strain (Figs. 3, 4) which likely increase ECQ and drug uptake which is account for the strain's susceptibility (Fig. 1). In fact, the null mutation of ERG6 renders the yeast strain increased sensitivity to various drugs ${ }^{51}$.

In yeast and fungi, sterols are synthesized in the endoplasmic reticulum (ER) and then transported to other cell membranes via non-vesicular lipid transport ${ }^{52}$. Excess free sterols in the ER are cytotoxic; however, they could be detoxified by neutral lipid synthetic enzymes and stored in the form of LDs ${ }^{53,54}$. Despite LDs being known for having a role in the storage of neutral lipids, they also play vital roles in lipid metabolism and homeostasis with important links to the dysfunction of LD-associated diseases in humans ${ }^{55}$. Furthermore, Erg6p is involved in the formation of lipid organelles or LDs in the neutral form of lipids ${ }^{53,56}$. An interatomic map of protein-protein contacts of LDs with mitochondria and peroxisomes also reveals the involvement of LD proteins Erg6p and Pet10p in 75\% of interactions detected, suggesting a key role of Erg6p in coordinating cellular signalling and response through formation of $\mathrm{LDs}^{57}$. The protein-protein interaction of Erg6 $\mathrm{p}$ and mitochondrial NADH-cytochrome b5 reductase Mcrlp that is involved in outer membrane sorting has been reported ${ }^{57}$. This interaction suggests a connection between Erg6p and Mcr1p mediation of LD-assisted transfer of substrates in the different sub-mitochondria ${ }^{57}$.

Treatment with a squalene monooxygenase inhibitor terbinafine results in increasing sensitivity of LD-less mutants ${ }^{43}$. The accumulation of the toxic lipid species squalene in yeasts occurs in LD-less mutants, as the excess squalene is normally stored in $\mathrm{LDs}^{42,43}$. Increasing ECQ concentrations mediate the accumulation of squalene which increases lethality in the LD-defective $\Delta$ erg6 strain but not in the wild-type strain with proper formation and function of LDs (Fig. 4). Measurement of intracellular squalene in LDs fraction could be further investigated. The defective squalene storage may result in lipotoxicity in yeast cells, leading to increased sensitivity in the mutant erg6 6 strain (Fig. 1). A number of reports described elevated of LDs in tumor tissues and, a recent study shows its association with drug resistance in lung cancer cells in which ER stress and apoptosis are shown to be suppressed ${ }^{58}$. Here, ECQ study also supports a connection between the role of highly dynamic organelle LDs and enhanced resistance to drugs.

Moreover, beside LDs, Erg6p is localized at various organelles namely the plasma membrane-associated endoplasmic reticulum and the mitochondrial outer membrane ${ }^{59-61}$. Its versatile setting suggested different and important roles of Erg6p in maintaining lipid and cell homeostasis, especially during cellular response to cytotoxic agents. As mentioned, Erg6p function is highly complex, involving over 1050 total interactions for 615 unique genes according to the SGD database. In attempt to explain the involvement of Erg6p during the ECQ treatment in the wild-type cells, increased LDs clustering where Erg6p likely locates suggests that Erg6p may move to various organelles according to LD trafficking route and finally get degraded via lipophagic process (Fig. 5). In contrast, in the ECQ-treated $\Delta$ erg6 strain, LDs are likely trapped and tethered to the ERs due to ECQ action in targeting actin (Figs. 3, 5).

The effect of ECQ is more evident in the sensitive $\Delta$ erg6 strain due to increased plasma membrane permeability (Figs. 1, 4). It is intriguing question how Erg6p might be functionally inhibited by ECQ? Our results support ECQ action on actin aggregation and a link between actin-mediated mobility of LDs. Cells may respond to ECQ by inducing the ERG6 and additional ERG gene expression (Fig. 2) to restore balance of lipids and maintain numerous critical functions of sterol in yeast cells. Depletion of Erg6p or deletion of ERG6 results in depletion of ergosterol, therefore compromising the function of fungal drug efflux transporters including Candida Cdr1p and S. cerevisiae multi-drug transporter Pdr5 $\mathrm{p}^{28,54}$. Thus, ECQ treatment exacerbates the effect of ERG6 deletion on lipid and sterol composition and content (Fig. 4), compromising the function of key drug efflux pumps or membrane associated proteins.

Despite some unanswered questions, several studies support Erg6p connecting function in LDs mobility. For example, the antifungal drug miconazole that targets Erg11p has also been shown to induce changes in actin cytoskeleton and activates several genes, involved in membrane trafficking, endocytosis and regulation of actin cytoskeleton, required for miconazole tolerance ${ }^{62}$. Due to a tight regulation of actin cytoskeleton organization and lipid homeostasis, particularly on formation and distribution of LDs which Erg6p plays an essential role for protein recycling as well as proper intracellular and surface cell delivery ${ }^{63}$. Yeast membrane lipid imbalance has been shown to cause trafficking defects between different organelles ${ }^{64}$. The membrane lipid mutant cells have been found to display lower levels of actin cables, suggesting that the actin cytoskeleton is disrupted upon membrane lipid imbalance. Recent study also indicates roles for LDs biogenesis and microlipophagy in adaptation to phosphatidylcholine lipid imbalance in yeast ${ }^{65}$. They report that lipid imbalance triggers several defects including, mitochondria and ER morphology, localization, motility and inheritance of mitochondria. Yeast adapts through 
LDs biogenesis at ER aggregates in accompanied with increased level of TAG and relevant TAG biosynthetic enzymes. Our analysis of TAG and sterol content in ECQ treated cells also supports such adaptation (Fig. 4).

Finally, stress-induced LD enriched with ubiquitinated proteins are then destined for vacuolar degradation via a process resembles microautophagy. Additionally, another study also shows that the endosomal sorting complex that plays role in transport, membrane sealing, trafficking and autophagy negatively regulates degradation of Erg6p-associated protein lipid droplets under glucose restricted conditions ${ }^{66}$. Overexpression or inactivation of enzymes of ergosterol, phospholipid or sphingolipid biosynthesis affects cellular trafficking. Membrane fusion is also bypassed by increased sterols as shown by overexpression of ERG6, which promotes actin remodeling as part the membrane fusion mechanism ${ }^{67}$. Additionally, high accumulation of zymosterol in the $\Delta$ erg6 strain is also observed (Fig. 4) as previously reported by another study ${ }^{39}$ because Erg6p functions to convert zymosterol to fecosterol. Since zymosterol is a toxic sterol that interferes with the integrity and function of yeast cell membranes ${ }^{48}$, an increased level of zymosterol is partly responsible for the growth defects of the $\Delta$ erg6 strain following ECQ treatment (Fig. 1, 4). In fact, zymosterol that circulates within the cells is the key precursor of ergosterol in fungi and of cholesterol in humans. In human fibroblasts, zymosterol is converted to cholesterol solely in the rough ER. Little or no zymosterol or cholesterol accumulates in the ER in vivo, because newly synthesized zymosterol moves to the plasma membrane without a detectable lag or about twice as fast as cholesterol ${ }^{60}$. Since ECQ treatment results in replacement of membrane sterol from ergosterol to zymosterol (Fig. 4), combinatorial treatment using antifungal ECQ and an inhibitor of the fungal-specific enzyme Erg6p may enhance drug sensitivity, offering a potential therapeutic approach in targeting pathogenic yeasts and fungi. Interestingly, Erg6p is the one of enzymes in ergosterol biosynthesis that are not involved in the cholesterol biosynthetic pathway. As mentioned Erg6p is responsible for the conversion of zymosterol to fecosterol and it is currently a promising and critical target for new antifungal drugs. Some inhibitors of Erg6p have been reported, such as azasterols; however, their use is limited due to the undesirable inhibition of the human enzyme 24-sterol reductase, which causes adverse toxic effects ${ }^{68,69}$.

Indeed, a correlation between the organization of the actin cytoskeleton and sterol synthesis has been also implicated in cell proliferation. To explore the benefits of ECQ, our previous work shows that ECQ enhances antifungal action of azole drugs and lowers dosage used of ECQ by approximately 10-50 times when combined with some azoles ${ }^{23}$. Although most studies on cytochalasins are explored in mammalian cell lines, effect of cytochalasin B and D at concentrations of 5 and $1 \mu \mathrm{g} / \mathrm{ml}$ is reported to complete actin disruption ${ }^{33}$. Much lower concentrations required as compared to yeast cells. Effects of cytochalasans B, D and E and novel phenochalasins are also found on cytosolic lipid droplet formation and neutral lipid synthesis as well as their ability to inhibit cholesteryl ester (CE) synthesis in mouse peritoneal macrophages with IC50 values ranging between 0.1 and 20 $\mu \mathrm{M}^{70}$. Additionally, cytochalasins effects on sterol metabolism and plasma membrane integrity are shown. For examples, intracellular uptake and esterification of cholesterol are blocked by cytochalasin D in human monocyte-derived macropages ${ }^{71}$. The actin cytoskeleton is important for the stimulation of cholesterol esterification by atherogenic lipoproteins in macrophages. Cytochalasin D treatment of macrophages also inhibits the ability of acetyl-low density lipoprotein to stimulate cholesterol esterification ${ }^{72}$. Further, inhibition of plasma membrane cholesterol internalization by Cytochalasin D inhibitor causes dose-dependent inhibition of steroid hormone synthesis in the MA-10 cells ${ }^{73}$. Therefore, appropriated dose of cytochalasins used in therapeutic treatments shall be clinically investigated. Optimization of ECQ production will be required for future experiments and commercially availability. Media optimization has been reported for improving ECQ production which provides approximately 4.4 -fold increase of yield ${ }^{74}$. Work is in progress to increase production of ECQ.

Importantly, some studies report benefits of cytochalasins in cancer treatments including increased the life expectancy and prolonged survival of mice challenged with leukemias ${ }^{75}$. Thus, in addition to their fungicidal activity, cytochalasins have unique antineoplastic activity with promising potential as a novel chemotherapeutic agent $^{76}$. Interestingly, squalene epoxidase is the not only target of fungicides but recently also gains increasing interest as a new target of anti-cancer drugs ${ }^{77}$. Cancer-associated small integral membrane open reading frame 1 (CASIMO1) is a known oncogene that is overexpressed predominantly in breast cancer patient samples. CASIMO1 contributes to the proper formation of the actin filament network and interacts squalene epoxidase, a major enzyme in cholesterol synthesis, of the mevalonate (MVA) pathway ${ }^{78}$. Recently, another study also shows that the cholesterol-independent feedback mechanism of sterol regulatory element-binding protein 1 (SREBP1) regulation is mediated by isoprenoids in regulating cell homoeostasis ${ }^{79}$. Our study in the model yeast $S$. cerevisiae and other recent findings in mouse and Drosophila models ${ }^{80-83}$ have unveiled a novel role of functional metabolites with potential implications in human health and disease.

In conclusion, the effects of the cytochalasin ECQ to disrupt actin depolymerization, a process critically affect LDs mobility on cytoskeleton network, and alter sterol metabolism are shown. ECQ-mediated disruption of actin function, results in gigantic formation of LDs clusters especially in the $\Delta$ erg6 mutant since LDs are transported directly along actin filaments ${ }^{84}$. Given a vital role of LDs on various life activities, including membrane homeostasis, investigation on membrane integrity or abnormal membrane microdomains is warranted.

\section{Materials and methods}

Xylaria culture and extraction. Xylaria sp. BCC1067 was obtained from the BIOTECH Culture Collection (BCC culture 6,200,032,292); National and Technology Development Agency, Bangkok, Thailand). Cultivation of Xylaria sp. BCC 1067 was done according to the method by Phonghanpot et al. ${ }^{85}$, with some modifications, as described by Somboon et al. ${ }^{22}$ Xylaria sp. BCC 1067 was grown on solid media containing $1.5 \%(\mathrm{w} / \mathrm{v})$ malt extract broth (MEB, OXOID, Oxoid Ltd., UK) and $2 \%(\mathrm{w} / \mathrm{v})$ agar (HIMEDIA, India) then transferred to fresh liquid MEB and allowed to grow for 25 days at $25^{\circ} \mathrm{C}$ without shaking. After, the culture was separated and 


\begin{tabular}{|c|c|c|}
\hline Strain & Genotype & Reference \\
\hline BY4742 & MATa his $3 \Delta 1$ leu $2 \Delta 0$ lys $2 \Delta 0$ ura $3 \Delta 0$ & Open Biosystems \\
\hline$\Delta u p c 2$ & 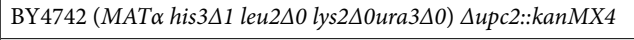 & Open Biosystems \\
\hline$\Delta h m g 1$ & 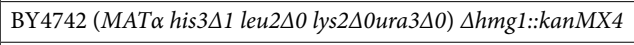 & Open Biosystems \\
\hline$\Delta s u t 1$ & BY4742 (MATa his3 $\Delta 1$ leu2 $\Delta 0$ lys $2 \Delta 0$ ura $3 \Delta 0) \Delta s u t 1:: k a n M X 4$ & Open Biosystems \\
\hline$\Delta e r g 4$ & 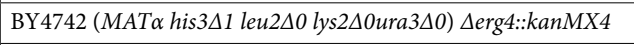 & Open Biosystems \\
\hline$\Delta e r g 5$ & 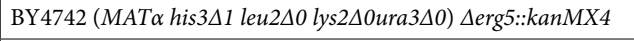 & Open Biosystems \\
\hline$\Delta e r g 6$ & 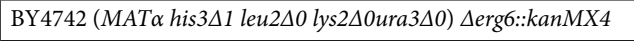 & Open Biosystems \\
\hline$\Delta e r g 28$ & 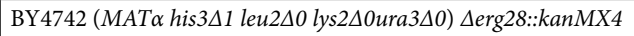 & Open Biosystems \\
\hline
\end{tabular}

Table 1. Genotypes S. cerevisiae wild-type and deletion mutant strains used in this study.

the cultural media fraction was then extracted with ethyl acetate (QREC, New Zealand) and dried under rotary evaporation. The dried crude extract was freshly dissolved with methanol prior to use.

Isolation of 19,20-epoxycytochalasin Q from the Xylaria sp. BCC 1067 extract. The isolation of bioactive compounds was described by Elias et al., with modifications ${ }^{86}$. One gram of crude Xylaria extract obtained from the cultural media fraction was subjected to silica gel column chromatography $(\varnothing 2.5 \mathrm{~cm} \times 25 \mathrm{~cm})$. The column was eluted with $\mathrm{CH}_{2} \mathrm{Cl}_{2} / \mathrm{EtOAC} / \mathrm{MeOH}$ and yielded the following 5 fractions (F1-F5): (ratio of $\mathrm{CH}_{2} \mathrm{Cl}_{2}-\mathrm{EtOAc}(5: 95,0: 100 \mathrm{v} / \mathrm{v}) 100 \mathrm{ml}$, EtOAc $100 \mathrm{ml}$ and ratio of EtOAc-MeOH $\left.(90: 10, \mathrm{v} / \mathrm{v}) 50 \mathrm{ml}\right)$. The pure ECQ compound was found in fraction F3, and F3 was then isolated further. The fraction F3 was subjected to silica gel column chromatography $(\varnothing 2 \mathrm{~cm} \times 25 \mathrm{~cm})$ using gradient $\mathrm{CH}_{2} \mathrm{Cl}_{2}-\mathrm{EtOAc}$. The fraction $\mathrm{F} 3$ was eluted with a gradient of solvent, as described, to give 9 fractions (S1-S9). The ECQ was found in fraction S5 that is further isolated by silica gel column chromatography $(\varnothing 1.5 \mathrm{~cm} \times 23 \mathrm{~cm})$ using a gradient of $\mathrm{CH}_{2} \mathrm{Cl}_{2}-\mathrm{EtOAc}_{\text {. }}$ The fraction S5 was eluted and, the fraction S5-1 was identified by High Performance Liquid Chromatography (HPLC) and determined by nuclear magnetic resonance (NMR) as 19,20-epoxycytochalasin Q.

Susceptibility assays of yeast deletion and ERG overexpression strains. The susceptibility of $S$. cerevisiae yeast deletion strains (Table 1) was testes against fluconazole, Xylaria sp. BCC 1067 extract, or ECQ using the microdilution reference method from the National Committee for Clinical Laboratory Standards ${ }^{87}$, with modifications according to Somboon et al. ${ }^{22}$. Yeast strains were treated with Xylaria extract or ECQ or fluconazole in 96 -well plate and incubated at $150 \mathrm{rpm}$ and $30^{\circ} \mathrm{C}$ for $24 \mathrm{~h} . \mathrm{OD}_{600}$ of cells were measured by using an automated microplate reader (M965+; METERTECH, Taipei, Taiwan). Normalized growth was calculated from normalized of $\mathrm{OD}_{600}$ of treated cells compared to untreated cells. Then, $3 \mu$ of cells were spotted on YPD agar plates and incubated for $48 \mathrm{~h}$ to observe cell survival. The standard culture media YPD (HIMEDIA, India) was utilized for MIC and MFC determinations. In addition, the susceptibility of yeast strains OVER-ERG1, OVER-ERG6, OVER-ERG11 and BY4742 + empty vector was investigated against ECQ using the microdilution reference method from the National Committee for Clinical Laboratory Standards ${ }^{87}$, with modifications. Yeast strains were cultured overnight under YPDG and adjusted to $\mathrm{OD}_{600}$ of 0.001 and regrown in YPG to induce expression of gene under GAL1 promotor. In 96-well plates, yeast cells were treated with different concentrations of ECQ and incubated at $30^{\circ} \mathrm{C}$ with shaking at $150 \mathrm{rpm}$ for $36 \mathrm{~h} . \mathrm{OD}_{600}$ of cells were measured by using an automated microplate reader (M965+; METERTECH, Taipei, Taiwan). Normalized growth was calculated from normalized of $\mathrm{OD}_{600}$ of ECQ-treated cells compared to untreated cells. Then, cells from micro-dilution assays were directly spotted $\left(10^{0}\right.$ dilution $)$ or 1000 times diluted $\left(10^{-3}\right.$ dilution $)$ on YPD plates to examine the survivability.

Gene induction and quantitative real-time polymerase chain reaction (qRT-PCR). The $S$. cerevisiae wild-type strain BY4742 was cultured in YPD at $30^{\circ} \mathrm{C}$ with shaking overnight. The yeast cells were measured and adjusted at an optical density $\left(\mathrm{OD}_{600}\right)$ of 0.1 for the starter cell. The culture was incubated until obtaining an $\mathrm{OD}_{600}$ of about 0.5 . Then, $4 \mu \mathrm{g} / \mathrm{ml}$ of ketoconazole or $500 \mu \mathrm{g} / \mathrm{ml}$ of Xylaria extract or $500 \mu \mathrm{g} / \mathrm{ml} \mathrm{of} \mathrm{ECQ} \mathrm{was}$ applied to cell culture for gene induction for $2 \mathrm{~h}$. Total RNAs were extracted as described by Schmitt et al. ${ }^{88}$. RNA was dissolved by DEPC and purified using the RNeasy Mini Kit (QIAGEN, Hilden, Germany). The purified RNA was used to synthesize cDNA by using the qPCRBIO cDNA synthesis kit (PCRBIOSYSTEMS, UK). The qRTPCR assays were performed using a Real-Time PCR Detection System with a software for analysis. The reaction mixtures contained Universal qPCR Master Mix (NEB). Gene-specific oligonucleotides were used are shown (Table 2). The relative expression data were analyzed using the $2^{-\Delta \Delta \mathrm{Ct}}$ method.

Quantification of sterols via gas chromatography-mass spectrometry (GC-MS). Quantification of sterol components was described by Li et al. ${ }^{89}$. The yeast strains $\left(2 \times 10^{5} \mathrm{cells} / \mathrm{ml}\right.$ in YPD medium), treated or untreated with the Xylaria extract or ECQ. The wild type cells were treated with 1000 or $225 \mu \mathrm{g} / \mathrm{ml}$ of Xylaria extract or ECQ, respectively. While the $\Delta \operatorname{erg} 6$ cells were treated with 225 or $112 \mu \mathrm{g} / \mathrm{ml}$ of Xylaria extract or ECQ, respectively. Concentration at MIC/4 was used to treat $\Delta$ erg6 strain with the Xylaria extract or ECQ and wild type strain with ECQ. For wild-type cells, $1000 \mu \mathrm{g} / \mathrm{ml}$ of Xylaria extract was used for treatment because could 


\begin{tabular}{|l|l|}
\hline Primer & Sequence \\
\hline$U P C 2-F$ & CTCCTACGATCAAGAAGGAGC \\
\hline$U P C 2-R$ & GAGATTGCTGCTGCACTTG \\
\hline$E C M 22-F$ & CATCAGCGGTCCACGATA \\
\hline$E C M 22-R$ & TGTTACCGCACCTATTAGCG \\
\hline$E R G 1-F$ & ATTCCATACCCTTACAAGGCC G \\
\hline$E R G 1-R$ & CGTGCATAGGAGCAGGATTC \\
\hline$E R G 2-F$ & CCACTGAAGACCTGTTACAGG \\
\hline$E R G 2-R$ & ACCTGTGTGCCCTTCAGTA \\
\hline$E R G 3-F$ & AGAAGGTTCTACGGGCAGG \\
\hline$E R G 3-R$ & GGATAGCACTGACTGCCAA \\
\hline$E R G 4-F$ & CACGGTAAGGTTGCCCTAC \\
\hline$E R G 4-R$ & ACATGCGTTCGCGTACAA \\
\hline$E R G 5-F$ & CGTTGTCGATGTTGCTGTGA \\
\hline$E R G 5-R$ & CTTCATGGCCATGTCTGC A \\
\hline$E R G 6-F$ & CCCAGCAAGAGAGATTGCA \\
\hline$E R G 6-R$ & AGGTCTTCGCTAACGAGGAC \\
\hline$E R G 9-F$ & GACGATATGTCCATCGAACAC G \\
\hline$E R G 9-R$ & GACAGTACACGTCGTAGTCG \\
\hline$E R G 11-F$ & GACCGTCCACCTCTAGTGT \\
\hline$E R G 11-R$ & CGTAAGCAGCTTCTGCTGA \\
\hline$E R G 1 O V E R \_F 1$ & CACTTAGGATCCATGTCTGCTG \\
\hline$E R G 1 O V E R \_R 1$ & CTGCCGCTCGAGTTAACCAATC \\
\hline$E R G 6 O V E R \_F$ & CGAGGGTACATGGCGGATCCATGAGTGAAACAGAATTGAG \\
\hline$E R G 1 O V E R \_R$ & CTAGCAACGATCGGCTCGAGTTATTGAGTTGCTTCTTGGG \\
\hline$E R G 11 O V E R \_F$ & GGCATTAGCGTGCGGATCCATGTCTGCTACCAAGTCAATC \\
\hline$E R G 11 O V E R \_R$ & GTCGGATGCGGCTCGAGTTAGATCTTTTGTTCTGGATTTC \\
\hline & \\
\hline
\end{tabular}

Table 2. Primers used in the real-time RT-PCR and plasmid construction.

not find MIC from susceptibility test. For GC-MS, cells were grown for $24 \mathrm{~h}$ at $30^{\circ} \mathrm{C}$ with shaking. Cells were then collected and resuspended in $40 \%$ alcoholic $\mathrm{KOH}$. The mixture was saponified by heating at $85^{\circ} \mathrm{C}$ for $1 \mathrm{~h}$ and allowed to cool to room temperature. The sterol was then extracted in petroleum ether and vaporized and analyzed by GC-MS (AGILENT 7890B). The gas chromatograph was equipped with a 5\% phenyl and $95 \%$ dimethylpolysiloxane column (length, $30 \mathrm{~m}$; inner diameter, $0.25 \mathrm{~mm}$; film thickness, $0.25 \mu \mathrm{m}$ ). The settings were as follows: initial GC temperature of $120^{\circ} \mathrm{C}$ for $4 \mathrm{~min}, 290^{\circ} \mathrm{C}$ for $3 \mathrm{~min}$ with gradient of $15^{\circ} \mathrm{C} / \mathrm{min}$; detector temperature, $340^{\circ} \mathrm{C}$; sample injection temperature, $320^{\circ} \mathrm{C}$; carrier gas, nitrogen gas; flow rate, $1 \mathrm{ml} / \mathrm{min}$. Sterols were identified from their retention times and specific mass spectrometric patterns.

Actin staining and fluorescence microscopy. The actin staining method was described by Gabriel et al..$^{90}$, with modifications. Wild-type $S$. cerevisiae and the $\Delta e r g 6$ strains were cultured in YPD at $30{ }^{\circ} \mathrm{C}$ with shaking overnight. The optical density $\left(\mathrm{OD}_{600}\right)$ of the cell culture was measured and adjusted to 0.1 as a starting $\mathrm{OD}$, and cells were incubated until obtaining an $\mathrm{OD}_{600}$ of 0.6 . Then, wild-type $S$. cerevisiae cells were treated with 1000 or $450 \mu \mathrm{g} / \mathrm{ml}$ of the Xylaria extract or ECQ, respectively, while the $\Delta$ erg6 cells were treated with 450 or $225 \mu \mathrm{g} / \mathrm{ml}$ of the Xylaria extract or ECQ, respectively. Then, the cells were collected at various time points of $0,2,4,6,12$, and $24 \mathrm{~h}$. Next, the cells were washed twice with Phosphate-buffered saline (PBS) buffer, incubated with $3.7 \%$ formaldehyde for $1 \mathrm{~h}$, stained with phalloidin-FITC labelled for $1 \mathrm{~h}$, and then washed twice with PBS buffer. The actin of the cells was examined with a confocal laser scanning microscope (FV10i-DOC).

Nile Red staining. The Nile Red staining method described by Rostron et al. ${ }^{91}$ was used with some modification. The yeast strains $\left(2 \times 10^{5}\right.$ cells/ml in YPD medium), treated or untreated with the Xylaria extract or ECQ, were grown for $24 \mathrm{~h}$ at $30^{\circ} \mathrm{C}$ with shaking. After that, $250 \mu \mathrm{L}$ of cells were transferred into sterile $1.5 \mathrm{~mL}$ tubes to be stained. $25 \mu \mathrm{L}$ of freshly prepared DMSO:PBS (1:1) and $5 \mu \mathrm{g} / \mathrm{mL}$ Nile Red in acetone were added, and the cells were incubated in the dark for $5 \mathrm{~min}$ at room temperature. The cells were washed twice with PBS and resuspended in $100 \mu \mathrm{L}$ of $10 \%(\mathrm{v} / \mathrm{v})$ formalin and fixed for $15 \mathrm{~min}$ in the dark at room temperature. The cells were washed twice with PBS and proceeded to imaging using a ZEISS Apotome.2 fluorescent microscope (ZEISS, Germany).

Quantification ofTAG. Quantification of TAG was investigated as previously described by Garaiová et al. ${ }^{92}$ with some modification. The yeast cells $\left(2 \times 10^{5}\right.$ cells $/ \mathrm{ml}$ in YPD medium), treated or untreated with the Xylaria extract or ECQ were collected and washed twice with DI water. Cells were disrupted by vortexing with glass 
beads and extracted by hot methanol $\left(30 \mathrm{~min}\right.$ at $\left.65^{\circ} \mathrm{C}\right)$. Then, cells were further incubated in chloroformmethanol (3:1) at room temperature. The organic phase containing lipids was collected and evaporated under $\mathrm{N}_{2}$ stream. Dry lipid residue was dissolved in toluene and investigated TAG content by High Performance Liquid Chromatography-Evaporative Light Scattering Detector (HPLC-ELSD).

Plasmid construction. To overexpress of ERG1, ERG6 and ERG11 gene in S. cerevisiae BY4742 (GenBank JRIR00000000). The pYES_ERG1, pYES_ERG6 and pYES_ERG11 plasmids were constructed in this study. The pYES2 plasmid was used as a vector which is the expression plasmid under GAL1 promotor in yeast expression system. In part of gene insert, the ERG1, ERG6 or ERG 11 genes were amplified from genomic DNA of S. cerevisiae by using High-Fidelity DNA Polymerase (NEW ENGLAND BIOLABS) and used primer that containing the sequence of restriction enzyme (BamHI and XhoI) (Table 2). The amplification condition was carried out as follows; $98^{\circ} \mathrm{C}$ for $30 \mathrm{~s}$, and the cycle of denaturation at $98^{\circ} \mathrm{C}$ for $10 \mathrm{~s}$, annealing at $45-50{ }^{\circ} \mathrm{C}$ for $30-60 \mathrm{~s}$, and extension at $72^{\circ} \mathrm{C}$ for $1-2 \mathrm{~min}$. The PCR product of individually gene was then digested with BamHI and XhoI, at $5^{\prime}$ and $3^{\prime}$ sites respectively, and ligated into the BamHI and XhoI sites of pYES2 to yield the plasmid pYES_ERG1, pYES_ERG6 and pYES_ERG11. Transformation was performed following by heat shock method ${ }^{93}$. The transformants were selected on LB agar plate containing $100 \mu \mathrm{g} / \mathrm{ml}$ of ampicillin and verified by restriction enzyme digestion and DNA sequencing. The pYES_ERG1, pYES_ERG6 and pYES_ERG11 constructed plasmids were individually transformed into S. cerevisiae BY4742 by LiAc/SS carrier DNA/PEG method ${ }^{94}$. The transformants were selected on synthetic defined (SD) yeast medium (without Uracil). The sequencing was performed and the expression was checked via qRT-PCR.

Statistical analysis. The results were presented as mean \pm SD and statistical analysis was performed by using one-way.

ANOVA, followed by Tukey's pairwise comparison by the SPSS Statistics 27.0 software (IBM, NY, USA). A $p$ value of $<0.05$ was considered as significantly different. At least three independent experiments were performed at least in triplicates.

Received: 26 September 2020; Accepted: 22 March 2021

Published online: 08 April 2021

\section{References}

1. Kett, D. H., Azoulay, E., Echeverria, P. M. \& Vincent, J.-L. Candida bloodstream infections in intensive care units: analysis of the extended prevalence of infection in intensive care unit study. Crit. Care Med. 39, 665-670 (2011).

2. Ostrosky-Zeichner, L., Marr, K. A., Rex, J. H. \& Cohen, S. H. Amphotericin B: time for a new" gold standard". Clin. Infect. Diseases 37, 415-425 (2003).

3. Kamiński, D. M. Recent progress in the study of the interactions of amphotericin B with cholesterol and ergosterol in lipid environments. Eur. Biophys. J. 43, 453-467 (2014).

4. Santos, J. R. A. et al. Dynamic interaction between fluconazole and amphotericin B against Cryptococcus gattii. Antimicrob. Agents Chemother. 56, 2553-2558 (2012).

5. Ghannoum, M. A. \& Rice, L. B. Antifungal agents: mode of action, mechanisms of resistance, and correlation of these mechanisms with bacterial resistance. Clin. Microbiol. Rev. 12, 501-517 (1999).

6. Vandeputte, P., Ferrari, S. \& Coste, A. T. Antifungal resistance and new strategies to control fungal infections. Int. J. Microbiol. 2012 (2011).

7. Soontorngun, N., Somboon, P. \& Watchaputi, K. in Non-conventional Yeasts: From Basic Research to Application (ed Andriy Sibirny) 453-476 (Springer, 2019).

8. Parks, L. W. \& Adams, B. G. Metabolism of sterols in yeast. CRC Crit. Rev. Microbiol. 6, 301-341 (1978).

9. Donald, K., Hampton, R. Y. \& Fritz, I. B. Effects of overproduction of the catalytic domain of 3-hydroxy-3-methylglutaryl coenzyme A reductase on squalene synthesis in Saccharomyces cerevisiae. Appl. Environ. Microbiol. 63, 3341-3344 (1997).

10. Polakowski, T., Stahl, U. \& Lang, C. Overexpression of a cytosolic hydroxymethylglutaryl-CoA reductase leads to squalene accumulation in yeast. Appl. Microbiol. Biotechnol. 49, 66-71 (1998).

11. Jordá, T. \& Puig, S. Regulation of ergosterol biosynthesis in saccharomyces cerevisiae. Genes 11, 795 (2020).

12. Mantzouridou, F. \& Tsimidou, M. Z. Observations on squalene accumulation in Saccharomyces cerevisiae due to the manipulation of HMG2 and ERG6. FEMS Yeast Res. 10, 699-707 (2010).

13. Sokolov, S., Trushina, N., Severin, F. \& Knorre, D. Ergosterol turnover in yeast: an interplay between biosynthesis and transport. Biochem. Mosc. 84, 346-357 (2019).

14. Kodedova, M. \& Sychrova, H. Changes in the sterol composition of the plasma membrane affect membrane potential, salt tolerance and the activity of multidrug resistance pumps in Saccharomyces cerevisiae. PLoS ONE 10, e0139306 (2015).

15. Scorzoni, L. et al. Antifungal therapy: New advances in the understanding and treatment of mycosis. Front. Microbiol. https://doi. org/10.3389/fmicb.2017.00036 (2017).

16. Aly, A. H., Debbab, A. \& Proksch, P. Fifty years of drug discovery from fungi. Fungal Divers. 50, 3 (2011).

17. Ko, H.-J., Song, A., Lai, M.-N. \& Ng, L.-T. Immunomodulatory properties of Xylaria nigripes in peritoneal macrophage cells of Balb/c mice. J. Ethnopharmacol. 138, 762-768 (2011).

18. Boonphong, S. et al. Multiplolides A and B, New Antifungal 10-Membered Lactones from Xylaria m ultiplex. J. Nat. Prod. 64, 965-967 (2001).

19. Adnan, M., Patel, M., Reddy, M. N. \& Alshammari, E. Formulation, evaluation and bioactive potential of Xylaria primorskensis terpenoid nanoparticles from its major compound xylaranic acid. Sci. Rep. 8, 1-12 (2018).

20. Chaichanan, J., Wiyakrutta, S., Pongtharangkul, T., Isarangkul, D. \& Meevootisom, V. Optimization of zofimarin production by an endophytic fungus, Xylaria sp. Acra L38. Braz. J. Microbiol. 45, 287-293 (2014).

21. Richardson, S. N. et al. Griseofulvin-producing Xylaria endophytes of Pinus strobus and Vaccinium angustifolium: evidence for a conifer-understory species endophyte ecology. Fungal Ecol. 11, 107-113 (2014).

22. Somboon, P. et al. Fungicide Xylaria sp. BCC 1067 extract induces reactive oxygen species and activates multidrug resistance system in Saccharomyces cerevisiae. Future Microbiol. 12, 417-440. https://doi.org/10.2217/fmb-2016-0151 (2017). 
23. Somboon, P. \& Soontorngun, N. An actin depolymerizing agent 19, 20-epoxycytochalasin Q of Xylaria sp. BCC 1067 enhanced antifungal action of azole drugs through ROS-mediated cell death in yeast. Microbiol. Res. 243, 126646 (2020).

24. Isaka, M. et al. Antiplasmodial compounds from the wood-decayed fungus Xylaria sp. BCC 1067. Planta Med. 66, 473-475 (2000).

25. Kodedová, M. \& Sychrová, H. Changes in the sterol composition of the plasma membrane affect membrane potential, salt tolerance and the activity of multidrug resistance pumps in Saccharomyces cerevisiae. PLoS ONE 10, e0139306. https://doi.org/10.1371/journ al.pone.0139306 (2015).

26. Hu, C. et al. Abnormal ergosterol biosynthesis activates transcriptional responses to antifungal azoles. Front. Microbiol. 9, 9 (2018).

27. Serratore, N. D. et al. A novel sterol-signaling pathway governs azole antifungal drug resistance and hypoxic gene repression in Saccharomyces cerevisiae. Genetics 208, 1037-1055 (2018).

28. Agarwal, A. K. et al. Genome-wide expression profiling of the response to polyene, pyrimidine, azole, and echinocandin antifungal agents in Saccharomyces cerevisiae. J. Biol. Chem. 278, 34998-35015 (2003).

29. Leber, R. et al. A novel sequence element is involved in the transcriptional regulation of expression of the ERG1 (squalene epoxidase) gene in Saccharomyces cerevisiae. Eur. J. Biochem. 268, 914-924 (2001).

30. Rybak, J. M. et al. Loss of C-5 sterol desaturase activity results in increased resistance to azole and echinocandin antifungals in a clinical isolate of Candida parapsilosis. Antimicrob. Agents Chemother. 61, e00651-e1617 (2017).

31. Slaughter, B. D., Smith, S. E. \& Li, R. Symmetry breaking in the life cycle of the budding yeast. Cold Spring Harb. Perspect. Biol. 1, a003384 (2009).

32. Vasicova, P., Lejskova, R., Malcova, I. \& Hasek, J. The stationary-phase cells of Saccharomyces cerevisiae display dynamic actin filaments required for processes extending chronological life span. Mol. Cell. Biol. 35, 3892-3908 (2015).

33. Kretz, R. et al. The effect of cytochalasans on the actin cytoskeleton of eukaryotic cells and preliminary structure-activity relationships. Biomolecules 9, 73 (2019).

34. Banan, A., Zhang, Y., Losurdo, J. \& Keshavarzian, A. Carbonylation and disassembly of the F-actin cytoskeleton in oxidant induced barrier dysfunction and its prevention by epidermal growth factor and transforming growth factor $\alpha$ in a human colonic cell line. Gut 46, 830-837 (2000).

35. Zhao, Y., MacGurn, J. A., Liu, M. \& Emr, S. The ART-Rsp5 ubiquitin ligase network comprises a plasma membrane quality control system that protects yeast cells from proteotoxic stress. Elife 2, e00459 (2013).

36. Daum, G., Lees, N. D., Bard, M. \& Dickson, R. Biochemistry, cell biology and molecular biology of lipids of Saccharomyces cerevisiae. Yeast 14, 1471-1510. https://doi.org/10.1002/(sici)1097-0061(199812)14:16\%3c1471::Aid-yea353\%3e3.0.Co;2-y (1998).

37. Veen, M., Stahl, U. \& Lang, C. Combined overexpression of genes of the ergosterol biosynthetic pathway leads to accumulation of sterols in Saccharomyces cerevisiae. FEMS Yeast Res. 4, 87-95 (2003).

38. Liu, G., Chen, Y., Færgeman, N. J. \& Nielsen, J. Elimination of the last reactions in ergosterol biosynthesis alters the resistance of Saccharomyces cerevisiae to multiple stresses. FEMS Yeast Res. 17. https://doi.org/10.1093/femsyr/fox063(2017).

39. Gaber, R. F., Copple, D. M., Kennedy, B. K., Vidal, M. \& Bard, M. The yeast gene ERG6 is required for normal membrane function but is not essential for biosynthesis of the cell-cycle-sparking sterol. Mol. Cell. Biol. 9, 3447-3456 (1989).

40. Parks, L. W., Smith, S. J. \& Crowley, J. H. Biochemical and physiological effects of sterol alterations in yeast-A review. Lipids 30, 227-230 (1995).

41. Bhattacharya, S., Esquivel, B. D. \& White, T. C. Overexpression or deletion of ergosterol biosynthesis genes alters doubling time, response to stress agents, and drug susceptibility in Saccharomyces cerevisiae. MBio 9, e01291-e11218 (2018).

42. Valachovic, M., Garaiova, M., Holic, R. \& Hapala, I. Squalene is lipotoxic to yeast cells defective in lipid droplet biogenesis. Biochem. Biophys. Res. Commun. 469, 1123-1128 (2016).

43. Csáky, Z. et al. Squalene lipotoxicity in a lipid droplet-less yeast mutant is linked to plasma membrane dysfunction. Yeast 37, 45-62. https://doi.org/10.1002/yea.3454 (2020).

44. Jarc, E. \& Petan, T. Focus: Organelles: Lipid droplets and the management of cellular stress. Yale J. Biol. Med. 92,435 (2019).

45. Ta, M. T. et al. Accumulation of squalene is associated with the clustering of lipid droplets. FEBS J. 279, 4231-4244 (2012).

46. Fei, W. et al. Fld1p, a functional homologue of human seipin, regulates the size of lipid droplets in yeast. J. Cell Biol. 180, 473-482 (2008).

47. Choudhary, V., El Atab, O., Mizzon, G., Prinz, W. A. \& Schneiter, R. Seipin and Nem1 establish discrete ER subdomains to initiate yeast lipid droplet biogenesis. J. Cell Biol. 219, e201910177 (2020).

48. Munn, A. L., Heese-Peck, A., Stevenson, B. J., Pichler, H. \& Riezman, H. Specific sterols required for the internalization step of endocytosis in yeast. Mol. Biol. Cell 10, 3943-3957 (1999).

49. Choudhary, V. \& Schneiter, R. Lipid droplet biogenesis from specialized ER subdomains. Microbial Cell 7, 218 (2020).

50. Landl, K. M., Klösch, B. \& Turnowsky, F. ERG1, encoding squalene epoxidase, is located on the right arm of chromosome VII of Saccharomyces cerevisiae. Yeast 12, 609-613 (1996).

51. Shah, N. \& Klausner, R. Brefeldin A reversibly inhibits secretion in Saccharomyces cerevisiae. J. Biol. Chem. 268, 5345-5348 (1993).

52. Jacquier, N. \& Schneiter, R. Mechanisms of sterol uptake and transport in yeast. J. Steroid Biochem. Mol. Biol. 129, $70-78$ (2012).

53. Jacquier, N. et al. Lipid droplets are functionally connected to the endoplasmic reticulum in Saccharomyces cerevisiae. J. Cell Sci. 124, 2424-2437 (2011).

54. Garbarino, J. et al. Sterol and diacylglycerol acyltransferase deficiency triggers fatty acid-mediated cell death. J. Biol. Chem. 284, 30994-31005 (2009).

55. Welte, M. A. \& Gould, A. P. Lipid droplet functions beyond energy storage. Biochimica et Biophysica Acta (BBA) Mol. Cell Biol. Lipids 1862, 1260-1272 (2017).

56. Cohen, S. in International Review of Cell and Molecular Biology Vol. 337 (ed Lorenzo Galluzzi) 83-110 (Academic Press, 2018).

57. $\mathrm{Pu}$, J. et al. Interactomic study on interaction between lipid droplets and mitochondria. Protein Cell 2, 487-496 (2011).

58. Jin, C. \& Yuan, P. Implications of lipid droplets in lung cancer: Associations with drug resistance. Oncol. Lett. 20, 2091-2104 (2020).

59. Zahedi, R. P. et al. Proteomic analysis of the yeast mitochondrial outer membrane reveals accumulation of a subclass of preproteins. Mol. Biol. Cell 17, 1436-1450 (2006).

60. Pichler, H. et al. A subfraction of the yeast endoplasmic reticulum associates with the plasma membrane and has a high capacity to synthesize lipids. Eur. J. Biochem. 268, 2351-2361 (2001).

61. Leber, R. et al. Dual localization of squalene epoxidase, Erglp, in yeast reflects a relationship between the endoplasmic reticulum and lipid particles. Mol. Biol. Cell 9, 375-386 (1998).

62. Thevissen, K. et al. Miconazole induces changes in actin cytoskeleton prior to reactive oxygen species induction in yeast. J. Biol. Chem. 282, 21592-21597 (2007).

63. Proszynski, T. J. et al. A genome-wide visual screen reveals a role for sphingolipids and ergosterol in cell surface delivery in yeast. Proc. Natl. Acad. Sci. 102, 17981-17986 (2005).

64. Woodman, S., Trousdale, C., Conover, J. \& Kim, K. Yeast membrane lipid imbalance leads to trafficking defects toward the Golgi. Cell Biol. Int. 42, 890-902 (2018).

65. Vevea, J. D. et al. Role for lipid droplet biogenesis and microlipophagy in adaptation to lipid imbalance in yeast. Dev. Cell 35, 584-599 (2015).

66. Zhang, A., Meng, Y., Li, Q. \& Liang, Y. The endosomal sorting complex required for transport complex negatively regulates Erg6 degradation under specific glucose restriction conditions. Traffic 21, 488-502 (2020). 
67. Tedrick, K., Trischuk, T., Lehner, R. \& Eitzen, G. Enhanced membrane fusion in sterol-enriched vacuoles bypasses the Vrplp requirement. Mol. Biol. Cell 15, 4609-4621. https://doi.org/10.1091/mbc.e04-03-0194 (2004).

68. Orenes Lorente, S. et al. Novel azasterols as potential agents for treatment of leishmaniasis and trypanosomiasis. Antimicrob. Agents Chemother. 48, 2937. https://doi.org/10.1128/AAC.48.8.2937-2950.2004 (2004).

69. Salci, T. P., Negri, M., Abadio, A. K. R., Svidzinski, T. I. E. \& Kioshima, É. S. Targeting Candida spp. to develop antifungal agents. Drug Discov. Today 23, 802-814 (2018).

70. Namatame, I., Tomoda, H., Arai, M. \& Omura, S. Effect of fungal metabolites cytochalasans on lipid droplet formation in mouse macrophages. J. Antibiot. 53, 19-25 (2000).

71. Kruth, H. S., Skarlatos, S. I., Lilly, K., Chang, J. \& Ifrim, I. Sequestration of acetylated LDL and cholesterol crystals by human monocyte-derived macrophages. J. Cell Biol. 129, 133-145 (1995).

72. Tabas, I., Zha, X., Beatini, N., Myers, J. N. \& Maxfield, F. R. The actin cytoskeleton is important for the stimulation of cholesterol esterification by atherogenic lipoproteins in macrophages. J. Biol. Chem. 269, 22547-22556 (1994).

73. Nagy, L. \& Freeman, D. A. Effect of cholesterol transport inhibitors on steroidogenesis and plasma membrane cholesterol transport in cultured MA-10 ley dig tumor cells. Endocrinology 126, 2267-2276 (1990).

74. Zhang, Y. et al. Improving the productivity of 19, 20-epoxy-cytochalasin Q in Xylaria sp. sof11 with culture condition optimization. Preparat. Biochem. Biotechnol. 46, 461-466 (2016).

75. Trendowski, M., Mitchell, J. M., Corsette, C. M., Acquafondata, C. \& Fondy, T. P. Chemotherapy with cytochalasin congeners in vitro and in vivo against murine models. Invest. New Drugs 33, 290-299 (2015).

76. Trendowski, M. Recent advances in the development of antineoplastic agents derived from natural products. Drugs 75, 1993-2016 (2015).

77. Brown, A. J., Chua, N. K. \& Yan, N. The shape of human squalene epoxidase expands the arsenal against cancer. Nat. Commun. 10, 1-4 (2019).

78. Polycarpou-Schwarz, M. et al. The cancer-associated microprotein CASIMO1 controls cell proliferation and interacts with squalene epoxidase modulating lipid droplet formation. Oncogene 37, 4750-4768 (2018).

79. Bertolio, R. et al. Sterol regulatory element binding protein 1 couples mechanical cues and lipid metabolism. Nat. Commun. 10, $1-11(2019)$.

80. Bernstein, B. W. \& Bamburg, J. R. Actin-ATP hydrolysis is a major energy drain for neurons. J. Neurosci. 23, 1-6 (2003).

81. Freed-Pastor, W. A. et al. Mutant p53 disrupts mammary tissue architecture via the mevalonate pathway. Cell 148, 244-258 (2012).

82. Paszek, M. J. et al. Tensional homeostasis and the malignant phenotype. Cancer Cell 8, 241-254 (2005).

83. Vu, V., Bui, P., Eguchi, M., Xu, A. \& Sweeney, G. Globular adiponectin induces LKB1/AMPK-dependent glucose uptake via actin cytoskeleton remodeling. J. Mol. Endocrinol. 51, 155-165 (2013).

84. Kilwein, M. D. \& Welte, M. A. Lipid droplet motility and organelle contacts. Contact 2, 2515256419895688 (2019).

85. Phonghanpot, S. et al. Biosynthesis of xyrrolin, a new cytotoxic hybrid polyketide/non-ribosomal peptide pyrroline with anticancer potential, in Xylaria sp. BCC 1067. Chembiochem Eur. J. Chem. Biol. 13, 895-903. https://doi.org/10.1002/cbic.201100746 (2012).

86. Elias, L. M. et al. The potential of compounds isolated from Xylaria spp as antifungal agents against anthracnose. Braz. J. Microbiol. 49, 840-847 (2018).

87. Fothergill, A. W. in Interactions of Yeasts, Moulds, and Antifungal Agents (ed G.S. Hall) 65-74 (Springer, 2012).

88. Schmitt, M. E., Brown, T. A. \& Trumpower, B. L. A rapid and simple method for preparation of RNA from Saccharomyces cerevisiae. Nucleic Acids Res. 18, 3091 (1990).

89. Li, S. et al. Eudesmane sesquiterpenes from Chinese liverwort are substrates of Cdrs and display antifungal activity by targeting Erg6 and Erg11 of Candida albicans. Bioorg. Med. Chem. 25, 5764-5771 (2017).

90. Gabriel, M., Horký, D., Svoboda, A. \& Kopecká, M. Cytochalasin D interferes with contractile actin ring and septum formation in Schizosaccharomyces japonicus var. versatilis. Microbiology 144, 2331-2344 (1998).

91. Rostron, K. A. \& Lawrence, C. L. in Histochemistry of Single Molecules 219-229 (Springer, 2017).

92. Garaiová, M., Zambojová, V., Šimová, Z., Griač, P. \& Hapala, I. Squalene epoxidase as a target for manipulation of squalene levels in the yeast Saccharomyces cerevisiae. FEMS Yeast Res. 14, 310-323 (2014).

93. Van Die, I. M., Bergmans, H. E. \& Hoekstra, W. P. Transformation in Escherichia coli: studies on the role of the heat shock in induction of competence. Microbiology 129, 663-670 (1983).

94. Gietz, D., St Jean, A., Woods, R. A. \& Schiestl, R. H. Improved method for high efficiency transformation of intact yeast cells. Nucleic Acids Res. 20, 1425 (1992).

\section{Acknowledgements}

The authors are thankful to D.U. and K.A. (KMUTT, Thailand) for facility use. Special thanks to E.L. (University of Otago, New Zealand), A.P., N.P., P.S., S.C. and A.N. (KMUTT, Thailand) for technical assistance. This research project is supported by Thailand Science Research and Innovation (TSRI), Basic Research Fund: Fiscal year 2021 under Project Number 64A306000037 and Thailand Research Fund (TRF) to N.S. Finally, we are grateful for funding from Petchra Pra Jom Klao Doctoral Scholarship (KMUTT) and the student scholarship of National research council of Thailand (NRCT) to K.W., the program management unit for human resources \& institutional development, research and innovation (Grant Number B01F630003) to N.P.

\section{Author contributions}

Conceived and designed the study and experiments: K.W. and N.S. Performed the experiments: K.W. and N.P. Analyzed the data: K.W., P.S., K.R. and N.S. Contributed reagents/materials/analysis tools: K.R. and N.S. Wrote the paper: K.W. and N.S. All authors reviewed the manuscript.

\section{Competing interests}

The authors declare no competing interests.

\section{Additional information}

Supplementary Information The online version contains supplementary material available at https://doi.org/ 10.1038/s41598-021-87342-4.

Correspondence and requests for materials should be addressed to N.S.

Reprints and permissions information is available at www.nature.com/reprints. 
Publisher's note Springer Nature remains neutral with regard to jurisdictional claims in published maps and institutional affiliations.

(c) (i) Open Access This article is licensed under a Creative Commons Attribution 4.0 International License, which permits use, sharing, adaptation, distribution and reproduction in any medium or format, as long as you give appropriate credit to the original author(s) and the source, provide a link to the Creative Commons licence, and indicate if changes were made. The images or other third party material in this article are included in the article's Creative Commons licence, unless indicated otherwise in a credit line to the material. If material is not included in the article's Creative Commons licence and your intended use is not permitted by statutory regulation or exceeds the permitted use, you will need to obtain permission directly from the copyright holder. To view a copy of this licence, visit http://creativecommons.org/licenses/by/4.0/.

(C) The Author(s) 2021 